\title{
Chromosome Evolution of Octoploid Strawberry
}

Michael A. Hardigan ${ }^{1}$, Mitchell J. Feldmann ${ }^{1}$, Anne Lorant ${ }^{1}$, Randi Famula ${ }^{1}$, Charlotte Acharya $^{1}$, Glenn Cole $^{1}$, Patrick P. Edger ${ }^{2}$, and Steven J. Knapp ${ }^{1 *}$

*Corresponding author: Steven J. Knapp (sjknapp@ucdavis.edu)

${ }^{1}$ Department of Plant Sciences, University of California, Davis, Davis, California, 95616, USA

${ }^{2}$ Department of Horticulture, Michigan State University, East Lansing, Michigan, 48824, USA

\begin{abstract}
The allo-octoploid cultivated strawberry (Fragaria $\times$ ananassa) originated through a combination of polyploid and homoploid hybridization, domestication of an interspecific hybrid lineage, and continued admixture of wild species over the last 300 years. While genes appear to flow freely between the octoploid progenitors, the genome structures and diversity of the octoploid species remain poorly understood. The complexity and absence of an octoploid genome frustrated early efforts to study chromosome evolution, resolve subgenomic structure, and develop a single coherent linkage group nomenclature. Here, we show that octoploid Fragaria species harbor millions of subgenome-specific DNA variants. Their diversity was sufficient to distinguish duplicated (homoeologous and paralogous) DNA sequences and develop 50K and $850 \mathrm{~K}$ SNP genotyping arrays populated with co-dominant, disomic SNP markers distributed throughout the octoploid genome. Whole-genome shotgun genotyping of an interspecific segregating population yielded $1.9 \mathrm{M}$ genetically mapped subgenome variants in 5,521 haploblocks spanning 3,394 cM in F. chiloensis subsp. lucida, and 1.6M genetically mapped subgenome variants in 3,179 haploblocks spanning 2,017 cM in $F$. $\times$ ananassa. These studies provide a dense genomic framework of subgenomespecific DNA markers for seamlessly cross-referencing genetic and physical mapping information, and unifying existing chromosome nomenclatures. Through comparative genetic mapping, we show that the genomes of geographically diverse wild octoploids are effectively diploidized and completely collinear. The preservation of genome structure among allo-octoploid taxa is a critical factor in the unique history of garden strawberry, where unimpeded gene flow supported both its origin and domestication through repeated cycles of interspecific hybridization.
\end{abstract}

Keywords: Fragaria, strawberry, polyploidy, genome evolution, domestication

\section{Introduction}

Interspecific homoploid hybridization and polyploidy-inducing hybrid events have been creative forces in plant genome evolution and speciation, acting as catalysts for de novo reorganization of chromosome structure (Alix et al., 2017; Jiao et al., 2011; Mandáková et al., 2019; McKain et al., 2016; Soltis et al., 2014b, 2014a, 2016; Vallejo-Marín et al., 2015; Wendel et al., 2016; Yakimowski and Rieseberg, 2014). The cultivated strawberry (Fragaria $\times$ ananassa Duchesne ex Rozier) is unique among domesticated crop species 


\section{Strawberry Chromosome Evolution}

because it arose through both processes. The chromosomes of octoploid garden strawberry $(2 n=8 x=56)$ evolved through a combination of ancient polyploidy, and repeated homoploid hybridization in the last three centuries (Darrow, 1966; Duchesne, 1766). The presence of duplicated (homoeologous) chromosomes in plants frequently leads to meiotic anomalies and associated chromosomal rearrangements, e.g., translocations and inversions, that reduce or eliminate gene flow between the donors and their polyploid offspring (Alix et al., 2017; Latta et al., 2019; Soltis et al., 2014b, 2014a). Similarly, meiotic mispairing in interspecific homoploid hybrids can lead to rearranged offspring chromosomes that differ from the chromosomes of one or both parents, resulting in reproductive barriers and hybrid speciation, as has been widely documented in sunflower (Helianthus) and other plants (Abbott et al., 2010; Barb et al., 2014; Burke et al., 2004; Rieseberg, 1997; Yakimowski and Rieseberg, 2014). However, reproductive barriers among octoploid Fragaria taxa remain essentially nonexistent, fueling the recurrence of interspecific homoploid hybridization in the origin, domestication, and modern-day breeding of $F . \times$ ananassa.

The modern $F . \times$ ananassa lineage traces its origin to extinct cultivars developed in western Europe in the 1700s. These cultivars were interspecific hybrids of nonsympatric wild octoploids from the New World: F. chiloensis subsp. chiloensis from South America and F. virginiana subsp. virginiana from North America (Darrow, 1966). Repeated introgression of alleles from diverse subspecific ecotypes of $F$. virginiana and $F$. chiloensis defined the later generations, coinciding artificial selection of horticulturally important traits among hybrid descendants in Europe and North America. Modern cultivars have emerged from 250 years of global migration and breeding within this admixed population (Darrow, 1966; Hardigan et al., 2018). Because alleles have been introgressed from up to eight octoploid subspecies, the genomes of modern $F$. $\times$ ananassa individuals are mosaics of their wild ancestors (Hardigan et al., 2018; Liston et al., 2014). Since the discovery of artificial hybrids at the Gardens of Versaille (Duchesne, 1766), natural interspecific hybrids ( $F$. $\times$ ananassa subsp. cuneifolia) were discovered in zones of sympatry between $F$. chiloensis subsp. pacifica and $F$. virginiana subsp. platypetala in western North America (Hancock Jr and Bringhurst, 1979; Luby et al., 1992; Salamone et al., 2013; Staudt, 1999). Neither cultivated F. $\times$ ananassa or wild $F . \times$ ananassa subsp. cuneifolia is reproductively isolated from their octoploid progenitors. Thus, genes appear to flow freely between the wild octoploid progenitors, and between the hybrids and their progenitors. While genomic rearrangements have been identified between homoeologous chromosomes, and relative to diploid species (Tennessen et al., 2014; van Dijk et al., 2014), the apparent absence of reproductive isolation implies that homoploid and polyploid hybridization events have not produced significant chromosome rearrangements among octoploid taxa. We hypothesized that the octoploids carry nearly collinear chromosomes tracing to the most recent common ancestor, despite one million years of evolution which produced multiple recognized species and subspecies.

The octoploid strawberry genome has been described as "notoriously complex" and an "extreme example of difficulty" for study (Folta and Davis, 2006; Hirakawa et al., 2014; Hirsch and Buell, 2013; Koskela et al., 2016). While GBS, GWAS, and NGS-reliant 


\section{Strawberry Chromosome Evolution}

applications are relatively straightforward in organisms with well-characterized reference genomes, such approaches were previously difficult or intractable in octoploid strawberry (Bassil et al., 2015; Liston et al., 2014; Tennessen et al., 2014; Vining et al., 2017). Genetic studies in octoploid strawberry previously relied on the genome of woodland strawberry ( $F$. vesca), an extant relative to one of four diploid subgenomes contained in F. × ananassa (Edger et al., 2017; Shulaev et al., 2011). For nearly a decade, the $F$. vesca genome was the only framework available for DNA variant discovery, gene discovery, genetic mapping, and genome-wide association studies in octoploid strawberry (Bassil et al., 2015; Davik et al., 2015; Pincot et al., 2018; Tennessen et al., 2014; Vining et al., 2017). The development of a chromosome-scale reference genome for $F . \times$ ananassa (Edger et al., 2019b) provided the physical framework needed to overcome previous barriers, and explore the organization and evolution of its progenitor genomes.

Here, we report the first study of chromosome evolution and genome structure in octoploid Fragaria using an octoploid genome-guided approach to DNA variant discovery and comparative mapping. We demonstrate the ability to differentiate duplicated (homoeologous) octoploid sequences using both NGS and array-based genotyping technologies when applied in conjunction with an octoploid reference genome. In doing so, we overcome a long-standing technical hurdle that has impeded efforts to study strawberry subgenome diversity and chromosome evolution. We estimated strawberry subgenomic diversity by whole-genome shotgun (WGS) sequencing of 93 genealogically and phylogenetically diverse $F$. $\times$ ananassa, $F$. chiloensis, and $F$. virginiana individuals. The frequency of unique WGS sequence alignment to the octoploid strawberry genome was characteristic of many diploid plant species (Hamilton and Buell, 2012; Lee and Schatz, 2012; Schatz et al., 2012; Treangen and Salzberg, 2012), and permitted the identification of millions of subgenome-specific DNA variants, effectively distinguishing homologous and homoeologous DNA sequences on every chromosome. Using the genetic diversity of $F$. $\times$ ananassa, we developed publicly available 50K and $850 \mathrm{~K}$ SNP arrays populated with subgenome anchored marker probes for octoploid genetic mapping and forward genetic studies. We then performed high-density genetic mapping of five octoploids representing $F . \times$ ananassa and both its progenitor species using a combination of WGS-based and array-based genotyping. Telomere-to-telomere genetic mapping of nearly every chromosome was enabled by the conserved disomic segregation observed in populations derived from wild species and $F . \times$ ananassa, underscoring the effective diploidization and meiotic stability of octoploid Fragaria. Comparative mapping of $F$. $\times$ ananassa and multiple subspecies of $F$. chiloensis and $F$. virginiana revealed the genome structures of the cultivated reference genotype (Camarosa) and its progenitor species were nearly identical.

The collinear and diploidized genomes of $F . \times$ ananassa and its progenitors support octoploid Fragaria as an evolutionary clade which achieved a relatively high degree of genome stability prior to the speciation and sub-speciation of $F$. chiloensis and $F$. virginiana. Strawberry's interspecific origin followed by successive hybridization throughout domestication is an unusual improvement pathway typically associated with 
perennial tree fruits, and frequently contributes to reproductive incompatibility or sterility in wide species hybrids (Hughes et al., 2007; Ladizinsky, 1985; Meyer and Purugganan, 2013; Miller and Gross, 2011). The preservation of genome structure among diverse octoploid Fragaria species and subspecies was likely essential to the unique history of the $F . \times$ ananassa lineage, which has undergone repeated cycles of homoploid hybridization without the formation of reproductive barriers or loss of fertility.

\section{Results \& Discussion}

\section{Subgenomic Diversity of Octoploid Fragaria}

We performed the first deep exploration of the homoeologous sequence diversity of octoploid Fragaria using the Camarosa v1.0 octoploid reference genome (Edger et al., 2019b) and a diversity panel of 93 strawberry individuals, including $47 \mathrm{~F} . \times$ ananassa samples, $24 F$. chiloensis samples, and 22 F. virginiana samples (Table S1). By incorporating subgenome specificity at the assembly level, previous barriers to copyspecific sequence alignment caused by strawberry's octoploid ancestral homology posed a less significant obstacle than repetitive DNA elements for diploid genomes such as maize (Hamilton and Buell, 2012; Lee and Schatz, 2012; Schatz et al., 2012; Treangen and Salzberg, 2012). The fraction of uniquely aligning (MapQ > 0) PE150 sequences averaged $83.2 \%$, and $90.9 \%$ of Camarosa PE250 sequences aligned uniquely (Figure S1), allowing comprehensive coverage and analysis of subgenomic diversity. Using genotype calling software FreeBayes and a series of hard-filters targeting unique sequence alignments, we identified 41.8M subgenomic SNP and indel mutations in $F . \times$ ananassa and its wild progenitors.

$F . \times$ ananassa has been described as "genetically narrow" due to the small number of founders in the pedigrees of modern cultivars (Dale and Sjulin, 1990; Sjulin and Dale, 1987; Stegmeir et al., 2010). Despite a small effective population size, our analyses show that massive genetic diversity has been preserved in $F$. $\times$ ananassa, with negligible difference between wild species and domesticated germplasm. The subgenome nucleotide diversity $(\pi)$ of $F . \times$ ananassa $(\pi=5.857 \times 10-3)$ was equivalent to wild progenitors $F$. chiloensis $(\pi=5.854 \times 10-3)$ and $F$. virginiana $(\pi=5.954 \times 10-3)$, and comparable to the sequence diversity of Zea mays landraces $(\pi=4.9 \times 10-3)$ and wild Zea mays spp. parviglumis progenitors ( $\pi=5.9 \times 10-3)$ (Hufford et al., 2012). Correlations of $F$. $\times$ ananassa, $F$. chiloensis, and F. virginiana diversity across the 28 octoploid chromosomes ranged from 0.93-0.97, showing the magnitude and distribution of genomic diversity are broadly conserved among octoploid taxa. This suggested that $F$. $\times$ ananassa was not strongly bottlenecked by domestication, or that its domestication bottleneck was mitigated by continued introgression of allelic diversity from wild subspecies (Darrow, 1966). We found that variance in the distribution of octoploid nucleotide diversity was influenced more significantly by subgenome ancestry than domestication and breeding (Table S2). The diploid F. vesca subgenome, dominant with respect to gene abundance and expression (Edger et al., 2019b), contained the least diverse homoeolog of every ancestral chromosome, while subgenomes derived from the ancestors of the extant Asian species ( $F$. iinumae and $F$. nipponica) contained 


\section{Strawberry Chromosome Evolution}

185

186

187

188

189

190

191

192

193

194

195

196

197

198

199

200

201

202

203

204

205

206

207

208

209

210

211

212

213

214

215

216

217

218

219

220

221

222

223

224

225

226

227

228

229

230 greater genetic diversity (Table S2). These differences show that subgenome dominance supports distinct levels of purifying selection and genetic diversity on different chromosomes of octoploid strawberry.

Because F. x ananassa was domesticated as an interspecific hybrid, individual performance is assumed to benefit from allelic diversity between $F$. chiloensis and $F$. virginiana. To support comparisons of strawberry heterozygosity with previously studied polyploid species, we estimated individual heterozygosity based on the genomic frequency of heterozygous nucleotides (nts) (potato metric) (Hardigan et al., 2017), and the frequency of heterozygosity at GBS-derived polymorphic sites (blueberry and cotton metric) (de Bem Oliveira et al., 2019; Page et al., 2013). Strawberry genomic heterozygosity ranged from $0.02-0.80 \%$ and averaged $0.46 \%$ genomic nts. This translated to an average of $11.1 \%$ heterozygosity at polymorphic marker sites. The most heterozygous octoploid genomes, White Carolina (1700s - 0.80\% nts), PI551736 (Peruvian landrace $-0.72 \% \mathrm{nts}$ ), Jucunda (mid-1800s - 0.70\% nts), and Ettersberg 121 (early 1900 s $-0.66 \%$ nts), were heirloom $F$. $\times$ ananassa types with more recent $F$. virginiana $x F$. chiloensis parentage. The average subgenomic heterozygosity of octoploid strawberry $(0.46 \% \mathrm{nts})$ was below diploid potato $(1.05 \% \mathrm{nts})$ and tetraploid potato (2.73\% nts) (Hardigan et al., 2017). The average heterozygosity of octoploid strawberry at GBS-derived polymorphic sites was below the allo-tetraploid cotton Agenome (13\% marker sites), above the cotton D-genome ( $<1 \%$ marker sites) (Page et al., 2013), and below autotetraploid blueberry (32.4\% marker sites) (de Bem Oliveira et al., 2019). Due to the presence of four ancestral homoeologs, traditional models of "fixed heterozygosity" applied to allopolyploid species (Comai, 2005; Obbard et al., 2006) assume an octoploid functional heterozygosity four-fold greater than subgenomic estimates. Under this model, recent $F$. virginiana $x F$. chiloensis hybrids such as White Carolina and Jucunda could be regarded as similarly heterozygous to autopolyploid species such as potato. However, assembly of the allo-octoploid strawberry genome uncovered rampant gene silencing, gene loss, and rearrangements relative to diploid ancestors (Edger et al., 2019b), eroding the conservation of ancestral allele function. The frequency of unique sequence alignment (Figure S1) and unbroken distribution of subgenomic variant detection (Figure S2) in our analysis underscore the extensive divergence of the four subgenomes. Thus, traditional polyploid allele dosage models assuming genome-wide fixed heterozygosity may be of limited usefulness for strawberry.

\section{Recombination Breakpoint Mapping of Octoploid Strawberry}

We used WGS sequence analysis and recombination breakpoint mapping of an octoploid strawberry population to explore the breadth of disomic variation as an indicator of bivalent pairing during meiosis. Several cytogenetic and DNA marker studies have proposed the occurrence of polysomy in strawberry (Fedorova, 1946; Lerceteau-Köhler et al., 2003; Senanayake and Bringhurst, 1967), while others suggest that octoploids are mainly disomic (Arulsekar and Bringhurst, 1981; Bringhurst, 1990; Byrne and Jelenkovic, 1976; Kunihisa et al., 2005). We performed low-coverage (4-8x) sequencing and subgenomic variant calling in a population $(n=189)$ derived from a cross between the $F$. $\times$ ananassa reference genotype Camarosa and the beach 


\section{Strawberry Chromosome Evolution}

strawberry ( $F$. chiloensis subsp. lucida) ecotype Del Norte. These parents were selected to provide a dense comparison of profiles of mappable diploid DNA variation in a natural octoploid and an artificial hybrid (reference genotype). Variant calling against the Camarosa v1.0 genome identified 3.7M subgenomic SNPs and indels inherited from 1.6M Camarosa heterozygous sites $(A B \times A A), 1.9 \mathrm{M}$ Del Norte heterozygous sites (AA $\times A B)$, and $0.2 \mathrm{M}$ co-heterozygous sites $(A B \times A B)$. We used the high-density variant data to perform haplotype mapping based on recombination breakpoint prediction, and evaluated segregation ratios of parental alleles across the 28 octoploid chromosomes.

We bypassed the computational demand of analyzing pairwise linkage across millions of DNA variants with missing data and genotyping errors by implementing the haplotype calling approach proposed by Huang et al. (2009) and Marand et al. (2017). Our approach performed a sliding-window analysis to predict crossover events, then estimated the consensus of co-segregating DNA variation between recombination breakpoints to reconstruct the representative genotypes of each haploblock, which were mapped as unique genetic markers. Using this approach, we mapped 1.9M Del Norte variants in 5,521 haploblocks spanning $3,393.86 \mathrm{cM}$, and $1.6 \mathrm{M}$ Camarosa variants in 3,179 haploblocks spanning 2,016.95 cM (Dataset S1). The paternal beach strawberry (Del Norte) map produced telomere-to-telomere coverage of the 28 octoploid chromosomes (Figure 1), providing the most comprehensive genetic map of an octoploid Fragaria genome to-date. The complete mapping of the extant homoeologs for all seven ancestral Fragaria chromosomes in Del Norte, and analysis of chromosomewide segregation distortion (Figure 2), showed that disomic recombination is ubiquitous in the genome of $F$. chiloensis. By contrast, less than $50 \%$ of the Camarosa genome could be mapped on chromosomes 1-1, 1-2, 2-4, 3-3, 5-2, 6-2, 6-3, 6-4, and 7-3 (Figure S3). We analyzed Camarosa heterozygosity and segregation distortion to determine whether the inability to map large segments of the genome was the result of polysomic recombination in F. $\times$ ananassa. This uncovered a near total loss of polymorphism in the unmapped regions of Camarosa (Figure 2), showing that incomplete mapping of $F$. $x$ ananassa resulted from depletion of heterozygosity in the hybrid genome, not polysomy. Sargent et al. (2012) previously reported extensive regions of homozygosity that affected mapping of $F . \times$ ananassa. Artificial selection pressure in commercially bred hybrids almost certainly accounts for the lower subgenomic heterozygosity of Camarosa relative to Del Norte, which does not support a critical role for genome-wide interspecific heterozygosity in driving cultivar performance.

\section{K Octoploid Screening Array}

We designed Affymetrix SNP genotyping arrays populated with subgenome-specific marker probes to enable genetic mapping, genome-wide association studies (GWAS), and genomic prediction in octoploid strawberry. DNA variants were selected for array design from the subgenomic diversity identified in the WGS panel (Figure 3). From the $90 \mathrm{M}$ total unfiltered variant sites, we extracted $45 \mathrm{M}$ unfiltered variants that segregated in $F . \times$ ananassa. To identify candidate DNA variants for marker design, we selected only biallelic SNPs above a low-diversity threshold ( $\pi \geq 0.05)$, excluded rare alleles (MAF $\geq 0.05$ ), required a VCF quality score $>20$, and excluded sites with $>15 \%$ missing data in the diversity panel. These filters yielded 8M subgenomic SNPs 


\section{Strawberry Chromosome Evolution}

segregating within the $F . \times$ ananassa subset of the diversity panel. We obtained 71-nt marker probes by extracting 35-nt sequences flanking each SNP site from the Camarosa v1.0 genome assembly. Marker probes for the 8M high-confidence SNP sites were then filtered to remove candidates that were problematic for array tiling. These included duplicate or near-duplicate probe sequences, probes that inherited ambiguous reference sequences (Ns), probes requiring double-tiling (A/T or $\mathrm{C} / \mathrm{G}$ alleles), and probes that Affymetrix scored as having low buildability. We retained $6.6 \mathrm{M}$ probes that targeted high-confidence $F$. $\times$ ananassa variants and were acceptable for array tiling.

We applied three selection criteria for determining a subset of $850 \mathrm{~K}$ marker probes for tiling a screening array: likelihood of probe binding interference by off-target variants, likelihood of off-target (non-single copy) probe binding, and physical genome distribution. The likelihood of probe binding interference was scored as the sum of nonreference allele frequencies for off-target variants in the 35-nt binding region adjacent to the target SNP. The likelihood of off-target probe binding was scored by performing BLAST alignment of the 71-nt probe sequences to the Camarosa v1.0 genome and quantifying the number of off-target alignments with query coverage above $90 \%$ and sequence identity above $90 \%$. We then iteratively parsed the Camarosa v1.0 genome using 10 kilobase $(\mathrm{kb})$ non-overlapping physical windows, extracting the best available marker from each window based on probe binding interference and off-target binding likelihoods, until reaching an $850 \mathrm{~K}$ probe threshold. We reserved $16 \mathrm{~K}$ positions for legacy markers from the iStraw SNP array (Bassil et al., 2015; Verma et al., 2016) that were polymorphic in a previous strawberry diversity study (Hardigan et al., 2018). The set of $850 \mathrm{~K}$ probe sequences was submitted to Affymetrix for constructing a screening array.

We genotyped a genetically diverse sample of 384 octoploid strawberry accessions to validate subgenome-specific marker performance on the $850 \mathrm{~K}$ screening array (Dataset S2). The sample fluorescence files were analyzed with the Axiom Suite in polyploid mode to generate marker clusters. Collectively, 446,644 of 850,000 marker probes produced QC-passing polymorphic genotype clusters showing disomic (allopolyploid) segregation. Among these, $78.3 \%$ were classified as "PolyHighResolution" in the Axiom terminology, producing diploid co-dominant genotype clusters $(A A, A B$, and $B B$ ) without detecting off-target allelic variation. Similarly, $18.8 \%$ of markers classified as "NoMinorHomozygote" in the Axiom terminology produced dominant genotype clusters in which heterozygotes $(A B)$ clustered with one of the homozygous genotype classes (AA or $\mathrm{BB}$ ). The remaining $2.9 \%$ of the markers showed detection of non-target alleles, and were classified as "OffTargetVariant" markers in the Axiom terminology. The HomRO statistic generated by the Axiom Suite estimates genotype cluster separation, and has been used as a metric to infer octoploid single-copy (i.e. subgenome or paralog specific) probe binding when values exceed 0.3 (Bassil et al., 2015). Based on this threshold, $74 \%$ of the QC-passing marker probes on the $850 \mathrm{~K}$ screening array exhibit single-copy binding, in addition to measuring subgenome-specific DNA variation (Figure S4). The complete set of 446,644 validated probes is made available for public use (Dataset S3). 


\section{Strawberry Chromosome Evolution}

\section{K Octoploid Production Array}

We selected 49,483 polymorphic marker probes from the $850 \mathrm{~K}$ validated probe set to build a 50K production array (Dataset S4). 5,809 LD-pruned $\left(r^{2}<0.50\right)$ marker probes were retained from the iStraw panel to support cross-referencing of octoploid QTL studies and linkage group nomenclatures. We targeted 2,878 genes based on Camarosa v1.0 functional annotations that indicated R-gene affiliated protein domains (Edger et al., 2019b) or homology to Fragaria vesca genes involved in flowering and fruit development expression networks (Hollender et al., 2014; Kang et al., 2013). Candidate genes were pre-allocated up to two markers (within $1 \mathrm{~kb}$ ) from the screening panel. We next selected a set of the most commonly segregating markers to support genetic mapping. We identified this set by selecting the marker with the highest pairwise diversity $(\pi)$ in $F . \times$ ananassa across non-overlapping $50 \mathrm{~kb}$ physical genome windows. The remainder of the $50 \mathrm{~K}$ array was populated by iteratively parsing the genome with $50 \mathrm{~kb}$ physical windows and selecting random QC-passing markers to provide an unbiased genome distribution. Both the $850 \mathrm{~K}$ and $50 \mathrm{~K}$ probe sets provide unbroken, telomere-to-telomere physical coverage of the 28 octoploid strawberry chromosomes (Figure S5). Within the $50 \mathrm{~K}$ probe set, $53 \%$ of the probes were located within genes, and $79 \%$ were located within $1 \mathrm{~kb}$ of a gene. The $50 \mathrm{~K}$ probe set was provided to Affymetrix for building the production array.

We screened 1,421 octoploid samples from multiple bi-parental populations and a large diversity panel on the $50 \mathrm{~K}$ production array. Collectively, 42,081 markers (85\%) successfully replicated QC-passing polymorphic genotype clusters when screened in the larger sample group. Of the 7,402 non-replicated markers, only $1 \%$ were excluded due to becoming monomorphic ("MonoHighResolution" Axiom class) or from increased missing data ("CallRateBelowThreshold" Axiom class). Sub-clustering and increased dispersion within the AA or BB genotype clusters ("AAvarianceX", "AAvarianceY", "BBvarianceX", "BBvarianceY" Axiom classes) accounted for $20 \%$ on non-replicated markers. The Axiom software provided no specific cause for failure for the remaining $79 \%$ of non-replicated markers. These results suggest that increasing the size and diversity of a genotyping population may affect the reproducibility of a fraction (15\%) of markers on the $50 \mathrm{~K}$ array, while a majority ( $85 \%$ ) are highly reproducible. The fraction of polymorphic co-dominant ("PolyHighResolution") markers increased from $78 \%$ on the $850 \mathrm{~K}$ screening array to $86 \%$ among reproducible markers $50 \mathrm{~K}$ production array.

\section{Genetic Mapping of Wild Octoploid Ecotypes}

We demonstrated that the 50K SNP array allows dense genetic mapping of heterozygous regions on all 28 chromosomes of $F . \times$ ananassa, $F$. chiloensis, and $F$. virginiana. Genetic mapping of $F$. chiloensis and $F$. virginiana provided telomere-totelomere physical representation of the 28 octoploid chromosomes, and near-complete representation within the individual wild maps (Dataset S1). We selected four octoploid parents from two outcrossing bi-parental populations genotyped on the 50K array for mapping. The first population was the Camarosa $x$ Del Norte $(F$. chiloensis subsp. lucida) population used for WGS recombination breakpoint mapping $(n=182)$. The second population was derived from a cross between $F$. virginiana subsp. virginiana accession PI552277 (female parent) and F. virginiana subsp. virginiana accession 


\section{Strawberry Chromosome Evolution}

PI612493 (male parent) $(n=96)$. The number of SNPs segregating in the octoploid parent genotypes varied considerably (Table S3). Camarosa contained the most segregating markers $(9,062)$, followed by PI552277 (5,575), PI612493 $(5,464)$, and Del Norte $(2,368)$. The larger number of markers segregating in Camarosa relative to the wild parent genotypes reflected the array design strategy, which targeted $F . \times$ ananassa diversity. The unbalanced representation of $F$. virginiana and $F$. chiloensis diversity was not expected, because genome-wide variant calls showed similar profiles of heterozygosity and nucleotide diversity in the wild progenitors, and showed that Del Norte was more heterozygous than Camarosa. The higher level of ascertainment bias against $F$. chiloensis diversity that resulted from probing $F . \times$ ananassa alleles supports previous findings that $F$. virginiana diversity is more prevalent in cultivated hybrids (Hardigan et al., 2018). We mapped heterozygous variant sites of the four parent octoploids using software ONEMAP (Margarido et al., 2007) to generate initial linkage groups and markers orders, and BatchMap (Schiffthaler et al., 2017) for marker reordering and genetic distance estimation. Despite the ascertainment bias for domesticated allelic diversity on the $50 \mathrm{~K}$ array, the relatively unbiased distribution of genomic heterozygosity in wild genotypes (Table S3) provided a more complete representation of the wild octoploid genomes than $F . \times$ ananassa (Camarosa) (Figure 4). Large homozygous regions that produced breaks in the Camarosa WGS haplotype map and 50K array map (chromosomes 1-1, 1-2, 2-4, 3-3, 5-2, 6-2, 6-3, 6-4, and 7-3) were clearly featured in the wild genetic maps (Figure 4). Camarosa contained an average of $11.7( \pm 6.8) \mathrm{SNPs} /$ megabase $(\mathrm{Mb})$ across 28 chromosomes, with as many as 25.2 SNPs/Mb (1-4) and as few as 0.8 SNPs/Mb (1-3), underscoring the scattered distribution of mappable subgenomic diversity in the commercial hybrid.

The wild octoploid maps revealed large $(\mathrm{Mb}+)$ chromosomal rearrangements relative to the Camarosa v1.0 physical genome on chromosomes 1-2, 1-4, 2-1, 2-3, 6-2, and 6-4. These rearrangements were conserved across the wild species genomes, and supported by corresponding regions represented in the Camarosa genetic map (1-2, 1$4,2-1$ ) (Figure S3), indicating intra-chromosomal scaffolding errors in the physical reference genome. The fraction of SNPs genetically mapping to non-reference chromosomes ranged from $1.5-1.9 \%$ in the four parents, with the highest fraction observed in Camarosa. This indicated minimal inter-chromosomal errors in the physical genome, and minimal inter-chromosomal marker discordance between octoploid progenitor species. Thus, the octoploid genetic maps provided no evidence of chromosome rearrangement among the octoploid species, or relative to $F . \times$ ananassa.

\section{Genome Structure of Ancestral Octoploids}

Previous studies have reported octoploid chromosome rearrangements relative to diploid Fragaria, potentially contributing to sex determination (Govindarajulu et al., 2015; Spigler et al., 2008, 2010; Tennessen et al., 2014), and there is phylogenetic evidence of chromosome exchanges among the four ancestral subgenomes (Edger et al., 2019b; Liston et al., 2014). However, there is no evidence for chromosome-scale structural variation between octoploid taxa. It remains unclear to what extent the structural variation of octoploid Fragaria reflects initial polyploid 'genome shock' associated with a common ancestor, or rather, ongoing mutations contributing to octoploid species 


\section{Strawberry Chromosome Evolution}

diversification. Through comparative mapping, we show that the genomes of diverse octoploid ecotypes contributing to the homoploid hybrid lineage of $F$. $\times$ ananassa are nearly completely collinear. We constructed genetic maps for two additional wild genotypes, F. chiloensis subsp. pacifica (SAL3) and F. virginiana subsp. platypetala (KB3) to capture a more diverse set of octoploid subspecific taxa. We aligned publicly available DNA capture sequences from an $F$. chiloensis subsp. chiloensis $\times F$. chiloensis subsp. pacifica population (GP33 $\times$ SAL3, $n=46$; Tennessen et al., 2014), and an $F$. virginiana subsp. platypetala $\times F$. virginiana subsp. platypetala population $(\mathrm{KB} 3 \times \mathrm{KB} 11, \mathrm{n}=46$; Tennessen et al., 2018) to the Camarosa v1.0 genome assembly, and predicted subgenome variant genotypes using FreeBayes. Genetic mapping of the DNA capture markers followed the protocol used for the 50K SNP datasets. Using the $50 \mathrm{~K}$ array linkage groups and DNA capture linkage groups (Dataset S1), we performed comparative mapping of four octoploid subspecies $-F$. chiloensis subsp. lucida (Del Norte), F. chiloensis subsp. pacifica (SAL3), F. virginiana subsp. platypetala (KB3), and F. virginiana subsp. virginiana (PI552277) - in ALLMAPS based on genetically mapped variant sites anchored to 50-kb physical genome windows in Camarosa v1.0. The chromosomes of the octoploid progenitor subspecies were completely syntenic (Figure 5 , Figure S6). Based on these results, large-scale chromosome rearrangements in octoploid Fragaria relative to the diploid ancestral genomes would have occurred early on, before the speciation of $F$. chiloensis and $F$. virginiana.

\section{Unification of Octoploid Chromosome Nomenclatures}

Previous octoploid genetic mapping studies relied on a variety of DNA marker technologies including early PCR-based assays (microsatellites, AFLPs), and later, technical ploidy reduction of sequence variants called against the diploid $F$. vesca genome based on DNA capture, GBS, or WGS-derived array probes (Bassil et al., 2015; Rousseau-Gueutin et al., 2008; Spigler et al., 2008, 2010; Tennessen et al., 2014, 2018; Vining et al., 2017). This diversity of marker genotyping strategies without information linking to the $F . \times$ ananassa physical genome has caused a proliferation of disconnected strawberry chromosome nomenclatures that may not accurately reflect the phylogenetic origins of its respective subgenomes. The Camarosa v1.0 reference genome provides an anchoring point for unifying the existing octoploid nomenclatures. We aligned all historic Fragaria microsatellite markers in the Rosacea Genomic Database (GDR) to the Camarosa v1.0 genome and anchored the Spigler et al. (2010) nomenclature to the Camarosa physical genome, which provided the corresponding linkage groups for anchoring the Tennessen et al. (2014) nomenclature to Camarosa v1.0. We then utilized legacy iStraw probes retained on the $50 \mathrm{~K}$ array to link the Sargent and van Dijk chromosome nomenclatures (Sargent et al., 2016; van Dijk et al., 2014) to the Camarosa v1.0 genome, which was scaffolded using the map published by Davik et al. (2015). In total, five of the most widely cited octoploid strawberry chromosome nomenclatures were unified in relation to the physical genome (Table 1). The existing octoploid nomenclatures each contained subgenome assignments that were incongruent with ancestral chromosomal origins determined by phylogenetic analysis of the physical genome (Edger et al., 2019b, 2019a), particularly with respect to the non-vesca and non-iinumae subgenomes. The unmasking of octoploid homoeologous chromosome lineages (Edger et al., 2019b, 2019a), and construction of 


\section{Strawberry Chromosome Evolution}

461

462

463

464

465

466

467

468

469

470

471

472

473

474

475

476

477

478

479

480

481

482

483

484

485

486

487

488

489

490

491

492

493

494

495

496

497

498

499

500

501

502

503

504

505

506

genetic maps showing complete collinearity among ancestral species, provide the foundation for a unified octoploid nomenclature reflecting the phylogenetic origins of its subgenomes.

\section{Conclusion}

Using the first octoploid genome-guided approach to subgenomic (diploid) variant discovery, we have demonstrated that the genomes of the octoploid progenitors of $F$. $\times$ ananassa are highly collinear and diploidized (Figure 5, Figure S6). Octoploid Fragaria taxa do not follow the common polyploid rule book for chromosome rearrangement (Alix et al., 2017; Chester et al., 2012; Cifuentes et al., 2010; Gaeta et al., 2007; Gaeta and Pires, 2010; Leitch and Leitch, 2008; Ramsey and Schemske, 2002; Renny-Byfield and Wendel, 2014; Wendel et al., 2016), but instead exhibit incredible karyotypic stability across biogeographically diverse subspecies. Strikingly, we did not observe any large-scale $(\mathrm{Mb}+)$ structural rearrangements (e.g., translocations or inversions) in the genomes of $F$. chiloensis, $F$. virginiana, or $F$. $\times$ ananassa (Figure 4). The broad conservation of chromosome structure across diverse progenitor taxa partly explains the absence of reproductive barriers and ease of gene flow among currently defined species and the domesticated hybrid lineage. In this regard, octoploid Fragaria species appear to be part of a minority among polyploid plants; however, similar examples of karyotypic stability have been described in monocots and dicots (Sun et al., 2017; VanBuren et al., 2019). Because of the ubiquity of polyploidy in angiosperms and the diversity of chromosome-restructuring outcomes along the pathway to diploidization, universal rules do not necessarily apply (Cifuentes et al., 2010; Le Comber et al., 2010; Renny-Byfield and Wendel, 2014; Wendel et al., 2016). The remarkable karyotypic stability and absence of chromosome rearrangements among octoploid Fragaria taxa are indicative of regular diploid meiotic behavior and suggest that homoeologous recombination has failed to disrupt the ancestral octoploid karyotype, which has been preserved over 0.4-2.1 M years of chromosome evolution and taxonomic diversification (Tennessen et al., 2014). The unique history of strawberry as a crop lineage, including its origin as an interspecific hybrid and frequent use of interspecific hybridization throughout domestication, was almost certainly supported by an uncommon stability of ancestral genome structure, and resulting ease of gene flow across octoploid genetic backgrounds.

The global importance and rapid commercial success of $F$. $\times$ ananassa over the last 250 years has been attributed to the interspecific homoploid hybrid component of heterosis (Rho et al., 2012; Shaw, 1995; Spangelo et al., 1971; Stegmeir et al., 2010). While some anecdotal evidence for heterosis exists in $F . \times$ ananassa, quantitative evidence is limited (Rho et al., 2012; Shaw, 1995; Spangelo et al., 1971; Stegmeir et al., 2010). Heterosis is an oft-cited advantage of polyploidy, where genomic heterozygosity is preserved by strict subgenomic recombination, the so-called "fixed heterosis" component of heterosis in allopolyploids (Comai, 2005). The $F . \times$ ananassa genome is riddled with ancient homoeologous exchanges (Edger et al., 2019b), a hallmark of intersubgenomic recombination in early generations. Neverthless, the genomes of presentday octoploid taxa appear to be highly diploidized. We observed disomic inheritance of 


\section{Strawberry Chromosome Evolution}

DNA variants across the genomes of the octoploids in the present study, and similar ranges of subgenomic heterozygosity for wild individuals and commercial hybrids. The success of $F . \times$ ananassa should not be solely attributed to "fixed heterosis" because neither octoploid progenitor species, which share the effects of fixed heterosis and show similar subgenomic heterozygosity, was commercially successful before the hybrid (Darrow, 1966; Finn et al., 2013). We hypothesize that interspecific complementation, a broader pool of potentially adaptive alleles, and masking of deleterious mutations could be more important than fixed heterosis in F. $\times$ ananassa (Alix et al., 2017; Comai, 2005).

We have shown that the purported complexity and previous intractability of octoploid strawberry genomics were largely associated with the technical challenge of distinguishing subgenome level variation from the broader pool of ancestral sequence homology. The use of an allo-octoploid reference genome addressed this problem by allowing variant calling based on unique sequence alignments to the respective subgenomes. While local subgenome homology could remain an issue, we identified a nearly continuous distribution of subgenome-specific variation spanning the octoploid genome by traditional short-read sequencing. With the design of the $850 \mathrm{~K}$ and $50 \mathrm{~K}$ arrays, this is now possible through high-density SNP marker genotyping, reducing bioinformatic requirements for octoploid strawberry research. In addition to expanding and validating the current molecular toolset, we have demonstrated that allopolyploid reference genomes facilitate the use of straightforward diploid approaches for genomic analysis and quantitative genetics of octoploid strawberry. In doing so, the results of this study help pave the way for molecular breeding of a historically difficult plant genome.

\section{Materials \& Methods}

\section{WGS Sequence Datasets}

We generated Illumina sequencing libraries for a diversity panel of 84 wild and domesticated octoploid genotypes (PE150), and the Camarosa reference genotype (PE250). Eight sequenced octoploid libraries (PE100) were obtained from the NCBI sequence read archive (SRA) (SRR1513906, SRR1513893, SRR1513905, SRR1513903, SRR1513892, SRR1513904, SRR1513867, SRR1513873), providing a total of 93 sample libraries in the diversity panel. We generated additional Illumina libraries (PE150) for 189 progeny of an $F$. $\times$ ananassa $\times$ F. chiloensis subsp. lucida mapping population (Camarosa $x$ Del Norte). Genomic DNA was extracted from immature leaf tissue using the E-Z 96 Plant DNA kit (Omega Bio-Tek, Norcross, GA, USA) with Proteinase $\mathrm{K}$ was added to the initial buffer, and RNase treatment delayed until lysate was removed from the cellular debris. An additional spin was added, and incubation steps were heated to $65 \mathrm{C}$ during elution. Libraries were prepared using the KAPA Hyper Plus kit using BIOO Nextflex adapters. DNA was sheared using the Covaris E220 and size selected for an average insert size of 300-nt using magnetic beads (Mag-Bind® RxnPure Plus, Omega Bio-tek). Libraries were pooled and sequenced on the Novaseq at UCSF for average $8 x$ genome coverage in the diversity panel, and $4-8 x$ coverage in the mapping population. DNA capture-based Illumina 
Strawberry Chromosome Evolution

sequences for the $F$. chiloensis subsp. lucida $\times F$. chiloensis subsp. pacifica population (GP33 x SAL3: $n=46$ ) described in Tennessen et al. (2014), and the $F$. virginiana subsp. platypetala $\times$ F. virginiana subsp. platypetala population (KB3 x KB11: $\mathrm{n}=46$ ) described in Tennessen et al. (2018) were downloaded from the NCBI SRA.

\section{Subgenomic WGS Variant Calling}

We predicted SNP and indel variants in the Camarosa v1.0 subgenomes using sequenced Illumina short-read libraries for the octoploid diversity panel, Camarosa $x$ Del Norte bi-parental population, and DNA capture sequences downloaded from the SRA. Short-read sequences were quality-trimmed with CutAdapt v1.8 using default parameters and a minimum Phred score of 25 . Trimmed short-reads were aligned to the Camarosa v1.0 genome assembly (Edger et al., 2019b) using BWA-mem v0.7.16, processed to mark optical and PCR duplicates using Picard Tools v2.18, and indelrealigned using GATK v3.8. Genomic variants were predicted based on uniquely mapped reads (MapQ > 20) using FreeBayes v1.2 and filtered with vcflib. For analysis of subgenomic variation, a set of hard-filters was applied to remove variants with low site quality (vcflib: QUAL > 40), low contribution of allele observations to site quality (vcflib: QUAL / AO > 10), low read coverage (vcflib: DP > 500), strand bias (vcflib: SAF $>0$ \& SAR > 0), read-placement bias (RPR > $1 \&$ RPL $>1$ ), unbalanced mapping quality of reference and alternate alleles (vcflib: $0.4 \leq$ [MQM / MQMR] $\leq 2.5$ ), unbalanced allele frequencies at heterozygous sites (vcflib: $0.2 \leq \mathrm{AB} \leq 0.8$ ), low end-placement probability score (EPP $\geq 3$ ), and low strand-bias probability score (vcflib: SRP $\geq 3$ \& SAP $\geq 3$ ). Sample genotypes were required to have individual read coverage $\geq 4$, and at least two reads and a minimum of 0.20 read observations supporting each allele.

\section{Octoploid Genomic Diversity}

We estimated octoploid genetic diversity metrics from a VCF file containing genotype calls for 45M filtered subgenomic SNPs and indels. We calculated the population-level diversity $(\pi)$ and per-sample heterozygosity of sequence variants using a custom perl script. Chromosomal and genome-wide population nucleotide diversity estimates were calculated as the sum of pairwise diversity for all variant sites divided by total non-gap (N) genomic nucleotides. Sample heterozygosity was calculated as the sum of heterozygous variant sites divided by total non-gap $(\mathrm{N})$ genomic nucleotides, and the fraction of total variant sites that were heterozygous.

\section{Array Design and Genotyping}

Unfiltered genomic variants were filtered to retain sites segregating in $F . \times$ ananassa cultivars. Cultivar variants were filtered to retain biallelic SNP sites with minor allele frequency $\geq 0.05$, marker diversity $\geq 0.05$, variant QUAL score $>20$, and missing data $<$ $15 \%$. Variants requiring 2-probe assays (A/T or $\mathrm{C} / \mathrm{G}$ ) were excluded. 71-nt marker probe sequences were obtained by retrieving 35-nt SNP flanking sequences from the Camarosa v1.0 assembly. Markers containing ambiguous sequences (Ns), or identical probes were excluded. A set of $6.6 \mathrm{M}$ probes was submitted to Affymetrix for scoring and recommendation based on strand, kmer uniqueness, and buildability. Probes were scored for likelihood of binding interference or non-specific binding based on off-target variant counts in the binding region, the sum of minor allele frequencies of interfering 


\section{Strawberry Chromosome Evolution}

variants, and counting off-target BLAST alignments (>90\% id, >90\% query length) in the genome. A final screening panel of 850,000 markers, including 16,000 iStraw probes (Bassil et al., 2015; Verma et al., 2016), was submitted to Affymetrix for constructing the $850 \mathrm{~K}$ screening array. A panel of 384 octoploid strawberry genotypes was screened on the $850 \mathrm{~K}$ array. Marker genotype clusters were scored using the Axiom Analysis Suite. Clustering was performed in "polyploid" mode with a marker call-rate threshold of 0.89 . Samples were filtered with a dQC threshold of 0.82 and QC CR threshold of 93. A subset of 49,483 probes was selected from polymorphic, QC-passing markers ("PolyHighResolution", "NoMinorHomozygote", "OffTargetVariant") on the 850K screening array to populate the 50K production array. 5,809 LD-pruned $\left(r^{2}<0.50\right)$ probes were pre-selected from the iStraw design, in addition to 47 probes associated with QTL for Fusarium oxysporum resistance and the Wasatch day neutral flowering locus (unpublished data). We assigned two markers per gene to a set of 2,878 genes located in expression networks related to flowering and fruit development (Hollender et al., 2014; Kang et al., 2013), or associated with R-gene domains. Non-overlapping 50 $\mathrm{kb}$ physical windows were parsed to select single markers containing the highest pairwise diversity in $F . \times$ ananassa genotypes. The remainder of the $50 \mathrm{~K}$ array was populated by iteratively parsing $50 \mathrm{~kb}$ physical windows to select random QC-passing markers for uniform genomic distribution. 1,421 octoploid samples, including the Camarosa $\times$ Del Norte mapping population $(n=182)$, and PI552277 x PI612493 mapping population $(n=96)$, were genotyped on the $50 \mathrm{~K}$ array and processed using the Axiom Analysis Suite using the same settings as the $850 \mathrm{~K}$ dataset.

\section{WGS Haplotype Linkage Mapping}

We used 1.6M female parent informative variant calls ( $A B \times A A)$, and $1.9 \mathrm{M}$ male parent informative variant calls $(A A \times A B)$ to generate haploblock markers for mapping genome-wide variant calls in the Camarosa $x$ Del Norte bi-parental population. Camarosa-informative and Del Norte-informative variant calls were divided into parentspecific marker sets, then split by chromosome. For each chromosome, we performed pairwise linkage disequilibrium (LD)-clustering of markers $(L D \geq 0.96)$ in an initial seed region containing the first 250 chromosome variants, to identify marker groups called in the same phase relative to the unphased Camarosa v1.0 genome. The genotype phase of the LD cluster containing the largest number of markers were selected as the "seed phase". A $50 \mathrm{~kb}$ sliding window was initiated in the seed region and moved across the chromosome, identifying downstream markers in negative LD with the seed phase and reversing the repulsion genotype calls, in order to phase the chromosome into an artificial backcross. If a phasing window skipped a physical region larger than $100 \mathrm{~kb}$ without markers, reached a window with fewer than 25 markers, or the average downstream marker LD fell below 0.90 , the chromosome was then fragmented at the breakpoint, seed-phase clustering repeated, and the sliding window reset for the subsequent downstream region. We used the software PhaseLD (Marand et al., 2017) with a 50-marker window and 1-marker step size to predict crossover events in the backcross-phased chromosome blocks. Window-specific variant calls lying between the predicted recombination breakpoints were used to generate consensus genotypes representing the haploblock. We mapped the haploblock markers using software ONEMAP (Margarido et al., 2007) and BatchMap (Schiffthaler et al., 2017) in outcross 
Strawberry Chromosome Evolution

645

mode. ONEMAP was used to bin co-segregating markers, calculate pairwise recombination fractions, determine optimal LOD thresholds, then cluster markers into linkage groups based on a LOD threshold of 8 , and maximum recombination fraction of 0.22 . Marker orders and genetic distances were re-estimated in parallel with BatchMap using a window of 50 markers, window overlap of 30, and ripple window of 6 markers.

\section{Array and DNA Capture Linkage Mapping}

We performed single-marker linkage mapping of populations genotyped using the $50 \mathrm{~K}$ array or DNA capture sequences because each contained fewer than 10,000 segregating markers per parent. Individual parent genotypes were mapped separately using their respective informative marker subsets. We filtered markers based on a chisquare test for segregation distortion ( $p$-value $<0.10$ ), and excluded markers with $>5 \%$ missing data. ONEMAP was used to bin co-segregating markers, calculate pairwise recombination fractions, determine optimal LOD thresholds, and cluster markers into linkage groups based on a LOD threshold of 8 , and maximum recombination fraction of 0.22 . Marker orders and genetic distances were re-estimated in parallel with BatchMap using a window of 20 markers, window overlap of 15, and ripple window of 6 markers.

\section{GDR Microsatellite Alignment}

We obtained the complete set of microsatellite primers developed in Fragaria species from the Rosaceae Genomics Database (GDR). Primers pairs were aligned to the Camarosa v1.0 genome in an orientation-aware manner using IPCRESS with a maximum amplicon fragment size of $500 \mathrm{bp}$ and allowing 1 mismatch per primer.

\section{Author Contributions}

$\mathrm{MAH}, \mathrm{PPE}$, and SJK contributed conception and design of the study; MAH, AL, and MJF performed the statistical analysis; RF, CA, and GC provided genetic material and collected, submitted DNA samples; MAH and SJK wrote the first draft of the manuscript; All authors contributed to manuscript revision, read and approved the submitted version.

\section{Acknowledgments}

We thank our collaborators at Affymetrix for constructing the $50 \mathrm{~K}$ and $850 \mathrm{~K}$ octoploid strawberry SNP genotyping arrays.

\section{Funding}

This research is supported by grants to S.J.K. from the United States Department of Agriculture (http://dx.doi.org/10.13039/100000199) National Institute of Food and Agriculture (NIFA) Specialty Crops Research Initiative (\#2017-51181-26833), and a United States Department of Agriculture NIFA postdoctoral fellowship (\#2018-6701227980).

\section{Data Availability}

The datasets generated for this study can be found in the NCBI Sequence Read Archive (https://www.ncbi.nlm.nih.gov/sra). 


\section{Strawberry Chromosome Evolution}

691

692

693

694

695

696

697

698

699

700

701

702

703

704

705

706

707

708

709

710

711

712

713

714

715

716

717

718

719

720

721

722

723

724

725

726

727

728

729

730

731

732

733

734

735

736

\section{References}

Abbott, R. J., Hegarty, M. J., Hiscock, S. J., and Brennan, A. C. (2010). Homoploid hybrid speciation in action. Taxon 59, 1375-1386.

Alix, K., Gérard, P. R., Schwarzacher, T., and Heslop-Harrison, J. S. (2017). Polyploidy and interspecific hybridization: partners for adaptation, speciation and evolution in plants. Ann. Bot. 120, 183-194.

Arulsekar, S., and Bringhurst, R. S. (1981). Genetic model for the enzyme marker PGI in diploid California Fragaria vesca L: Its variability and use in elucidating the mating system. J. Hered. 72, 117-120.

Barb, J. G., Bowers, J. E., Renaut, S., Rey, J. I., Knapp, S. J., Rieseberg, L. H., et al. (2014). Chromosomal evolution and patterns of introgression in Helianthus. Genetics 197, 969-979.

Bassil, N. V, Davis, T. M., Zhang, H., Ficklin, S., Mittmann, M., Webster, T., et al. (2015). Development and preliminary evaluation of a $90 \mathrm{~K}$ Axiom® SNP array for the allo-octoploid cultivated strawberry Fragaria× ananassa. BMC Genomics 16, 155.

Bringhurst, R. S. (1990). Cytogenetics and evolution in American Fragaria. HortScience $25,879-881$.

Burke, J. M., Lai, Z., Salmaso, M., Nakazato, T., Tang, S., Heesacker, A., et al. (2004). Comparative mapping and rapid karyotypic evolution in the genus Helianthus. Genetics 167, 449-457.

Byrne, D., and Jelenkovic, G. (1976). Cytological diploidization in the cultivated octoploid strawberry Fragariax ananassa. Can. J. Genet. Cytol. 18, 653-659.

Chester, M., Gallagher, J. P., Symonds, V. V., da Silva, A. V. C., Mavrodiev, E. V, Leitch, A. R., et al. (2012). Extensive chromosomal variation in a recently formed natural allopolyploid species, Tragopogon miscellus (Asteraceae). Proc. Natl. Acad. Sci. 109, 1176-1181.

Cifuentes, M., Grandont, L., Moore, G., Chèvre, A. M., and Jenczewski, E. (2010). Genetic regulation of meiosis in polyploid species: new insights into an old question. New Phytol. 186, 29-36.

Comai, L. (2005). The advantages and disadvantages of being polyploid. Nat. Rev. Genet. 6, 836.

Dale, A., and Sjulin, T. M. (1990). Few cytoplasm contribute to North American strawberry cultivars. HortScience 25, 1341-1342.

Darrow, G. M. (1966). The strawberry. History, breeding and physiology. Holt, Rinehart \& Winston, New York.

Davik, J., Sargent, D. J., Brurberg, M. B., Lien, S., Kent, M., and Alsheikh, M. (2015). A ddRAD based linkage map of the cultivated strawberry, Fragaria xananassa. PLoS One 10, e0137746.

de Bem Oliveira, I., Resende, M. F. R., Ferrão, L. F. V, Amadeu, R. R., Endelman, J. B., Kirst, M., et al. (2019). Genomic prediction of autotetraploids; influence of relationship matrices, allele dosage, and continuous genotyping calls in phenotype prediction. G3 Genes, Genomes, Genet. 9, 1189-1198.

Duchesne, A.-N. (1766). Histoire naturelle des fraisiers contenant les vues d'économie réunies à la botanique, et suivie de remarques particulières sur plusieurs points qui ont rapport à l'histoire naturelle générale, par M. Duchesne fils. chez Didot le jeune. 


\section{Strawberry Chromosome Evolution}

Edger, P. P., McKain, M. R., Yocca, A. E., Knapp, S. J., Qiao, Q., and Zhang, T. (2019a). Phylogenetic analyses and Fragaria iinumae genome support octopoid strawberry origin.

Edger, P. P., Poorten, T. J., VanBuren, R., Hardigan, M. A., Colle, M., McKain, M. R., et al. (2019b). Origin and evolution of the octoploid strawberry genome. Nat. Genet. 51, 541-547. doi:10.1038/s41588-019-0356-4.

Edger, P. P., VanBuren, R., Colle, M., Poorten, T. J., Wai, C. M., Niederhuth, C. E., et al. (2017). Single-molecule sequencing and optical mapping yields an improved genome of woodland strawberry (Fragaria vesca) with chromosome-scale contiguity. Gigascience 7, gix124.

Fedorova, N. J. (1946). Crossability and phylogenetic relations in the main European species of Fragaria. Compil. Natl. Acad. Sci. USSR 52, 545-547.

Finn, C. E., Retamales, J. B., Lobos, G. A., and Hancock, J. F. (2013). The Chilean strawberry (Fragaria chiloensis): Over 1000 years of domestication. HortScience $48,418-421$.

Folta, K. M., and Davis, T. M. (2006). Strawberry genes and genomics. CRC. Crit. Rev. Plant Sci. 25, 399-415.

Gaeta, R. T., and Pires, J. C. (2010). Homoeologous recombination in allopolyploids: the polyploid ratchet. New Phytol. 186, 18-28.

Gaeta, R. T., Pires, J. C., Iniguez-Luy, F., Leon, E., and Osborn, T. C. (2007). Genomic changes in resynthesized Brassica napus and their effect on gene expression and phenotype. Plant Cell 19, 3403-3417.

Govindarajulu, R., Parks, M., Tennessen, J. A., Liston, A., and Ashman, T. (2015). Comparison of nuclear, plastid, and mitochondrial phylogenies and the origin of wild octoploid strawberry species. Am. J. Bot. 102, 544-554.

Hamilton, J. P., and Buell, C. R. (2012). Advances in plant genome sequencing. Plant J. 70, 177-190.

Hancock Jr, J. F., and Bringhurst, R. S. (1979). Ecological differentiation in perennial, octoploid species of Fragaria. Am. J. Bot. 66, 367-375.

Hardigan, M. A., Laimbeer, F. P. E., Newton, L., Crisovan, E., Hamilton, J. P., Vaillancourt, B., et al. (2017). Genome diversity of tuber-bearing Solanum uncovers complex evolutionary history and targets of domestication in the cultivated potato. Proc. Natl. Acad. Sci. 114, E9999-E10008.

Hardigan, M. A., Poorten, T. J., Acharya, C. B., Cole, G. S., Hummer, K. E., Bassil, N., et al. (2018). Domestication of Temperate and Coastal Hybrids with Distinct Ancestral Gene Selection in Octoploid Strawberry. Plant Genome 11.

Hirakawa, H., Shirasawa, K., Kosugi, S., Tashiro, K., Nakayama, S., Yamada, M., et al. (2014). Dissection of the octoploid strawberry genome by deep sequencing of the genomes of Fragaria species. DNA Res. 21, 169-181.

Hirsch, C. N., and Buell, C. R. (2013). Tapping the promise of genomics in species with complex, nonmodel genomes. Annu. Rev. Plant Biol. 64, 89-110.

Hollender, C. A., Kang, C., Darwish, O., Geretz, A., Matthews, B. F., Slovin, J., et al. (2014). Floral transcriptomes in woodland strawberry uncover developing receptacle and anther gene networks. Plant Physiol. 165, 1062-1075.

Huang, X., Feng, Q., Qian, Q., Zhao, Q., Wang, L., Wang, A., et al. (2009). Highthroughput genotyping by whole-genome resequencing. Genome Res. 19, 1068- 


\section{Strawberry Chromosome Evolution}

1076.

Hufford, M. B., Xu, X., Van Heerwaarden, J., Pyhäjärvi, T., Chia, J.-M., Cartwright, R. A., et al. (2012). Comparative population genomics of maize domestication and improvement. Nat. Genet. 44, 808.

Hughes, C. E., Govindarajulu, R., Robertson, A., Filer, D. L., Harris, S. A., and Bailey, C. D. (2007). Serendipitous backyard hybridization and the origin of crops. Proc. Natl. Acad. Sci. 104, 14389-14394.

Jiao, Y., Wickett, N. J., Ayyampalayam, S., Chanderbali, A. S., Landherr, L., Ralph, P. E., et al. (2011). Ancestral polyploidy in seed plants and angiosperms. Nature 473, 97.

Kang, C., Darwish, O., Geretz, A., Shahan, R., Alkharouf, N., and Liu, Z. (2013). Genome-scale transcriptomic insights into early-stage fruit development in woodland strawberry Fragaria vesca. Plant Cell 25, 1960-1978.

Koskela, E. A., Sønsteby, A., Flachowsky, H., Heide, O. M., Hanke, M., Elomaa, P., et al. (2016). TERMINAL FLOWER 1 is a breeding target for a novel everbearing trait and tailored flowering responses in cultivated strawberry (Fragariax ananassa Duch.). Plant Biotechnol. J. 14, 1852-1861.

Kunihisa, M., Fukino, N., and Matsumoto, S. (2005). CAPS markers improved by cluster-specific amplification for identification of octoploid strawberry (Fragariax ananassa Duch.) cultivars, and their disomic inheritance. Theor. Appl. Genet. 110, $1410-1418$.

Ladizinsky, G. (1985). Founder effect in crop-plant evolution. Econ. Bot. 39, 191-199.

Latta, R. G., Bekele, W. A., Wight, C. P., and Tinker, N. A. (2019). Comparative linkage mapping of diploid, tetraploid, and hexaploid Avena species suggests extensive chromosome rearrangement in ancestral diploids. Sci. Rep. 9, 1-12.

Le Comber, S. C., Ainouche, M. L., Kovarik, A., and Leitch, A. R. (2010). Making a functional diploid: from polysomic to disomic inheritance. New Phytol. 186, 113122.

Lee, H., and Schatz, M. C. (2012). Genomic dark matter: the reliability of short read mapping illustrated by the genome mappability score. Bioinformatics 28, 20972105.

Leitch, A. R., and Leitch, I. J. (2008). Genomic plasticity and the diversity of polyploid plants. Science (80-. ). 320, 481-483.

Lerceteau-Köhler, E., Guerin, G., Laigret, F., and Denoyes-Rothan, B. (2003). Characterization of mixed disomic and polysomic inheritance in the octoploid strawberry (Fragariax ananassa) using AFLP mapping. Theor. Appl. Genet. 107, 619-628.

Liston, A., Cronn, R., and Ashman, T. (2014). Fragaria: a genus with deep historical roots and ripe for evolutionary and ecological insights. Am. J. Bot. 101, 1686-1699.

Luby, J. J., Hancock, J. F., and Ballington, J. R. (1992). Collection of native strawberry germplasm in the Pacific Northwest and Northern Rocky Mountains of the United States. HortScience 27, 12-17.

Mandáková, T., Pouch, M., Brock, J. R., Al-Shehbaz, I. A., and Lysak, M. A. (2019). Origin and Evolution of Diploid and Allopolyploid Camelina Genomes was Accompanied by Chromosome Shattering. Plant Cell, tpc-00366.

Marand, A. P., Jansky, S. H., Zhao, H., Leisner, C. P., Zhu, X., Zeng, Z., et al. (2017). 
Strawberry Chromosome Evolution

Meiotic crossovers are associated with open chromatin and enriched with Stowaway transposons in potato. Genome Biol. 18, 203.

Margarido, G. R. A., Souza, A. P., and Garcia, A. A. F. (2007). OneMap: software for genetic mapping in outcrossing species. Hereditas 144, 78-79.

McKain, M. R., Tang, H., McNeal, J. R., Ayyampalayam, S., Davis, J. I., dePamphilis, C. W., et al. (2016). A phylogenomic assessment of ancient polyploidy and genome evolution across the Poales. Genome Biol. Evol. 8, 1150-1164.

Meyer, R. S., and Purugganan, M. D. (2013). Evolution of crop species: genetics of domestication and diversification. Nat. Rev. Genet. 14, 840.

Miller, A. J., and Gross, B. L. (2011). From forest to field: perennial fruit crop domestication. Am. J. Bot. 98, 1389-1414.

Obbard, D. J., Harris, S. A., and Pannell, J. R. (2006). Simple allelic-phenotype diversity and differentiation statistics for allopolyploids. Heredity (Edinb). 97, 296.

Page, J. T., Huynh, M. D., Liechty, Z. S., Grupp, K., Stelly, D., Hulse, A. M., et al. (2013). Insights into the evolution of cotton diploids and polyploids from wholegenome re-sequencing. G3 Genes, Genomes, Genet. 3, 1809-1818.

Pincot, D. D. A., Poorten, T. J., Hardigan, M. A., Harshman, J. M., Acharya, C. B., Cole, G. S., et al. (2018). Genome-wide association mapping uncovers Fw1, a dominant gene conferring resistance to Fusarium wilt in strawberry. G3 Genes, Genomes, Genet. 8, 1817-1828.

Ramsey, J., and Schemske, D. W. (2002). Neopolyploidy in flowering plants. Annu. Rev. Ecol. Syst. 33, 589-639.

Renny-Byfield, S., and Wendel, J. F. (2014). Doubling down on genomes: polyploidy and crop plants. Am. J. Bot. 101, 1711-1725.

Rho, I. R., Woo, J. G., Jeong, H. J., Jeon, H. Y., and Lee, C. (2012). Characteristics of $\mathrm{F} 1$ hybrids and inbred lines in octoploid strawberry (Fragaria× ananassa Duchesne). Plant Breed. 131, 550-554.

Rieseberg, L. H. (1997). Hybrid origins of plant species. Annu. Rev. Ecol. Syst. 28, 359389.

Rousseau-Gueutin, M., Lerceteau-Köhler, E., Barrot, L., Sargent, D. J., Monfort, A., Simpson, D., et al. (2008). Comparative genetic mapping between octoploid and diploid Fragaria species reveals a high level of colinearity between their genomes and the essentially disomic behavior of the cultivated octoploid strawberry. Genetics 179, 2045-2060.

Salamone, I., Govindarajulu, R., Falk, S., Parks, M., Liston, A., and Ashman, T. (2013). Bioclimatic, ecological, and phenotypic intermediacy and high genetic admixture in a natural hybrid of octoploid strawberries. Am. J. Bot. 100, 939-950.

Sargent, D. J., Passey, T., Šurbanovski, N., Girona, E. L., Kuchta, P., Davik, J., et al. (2012). A microsatellite linkage map for the cultivated strawberry (Fragariax ananassa) suggests extensive regions of homozygosity in the genome that may have resulted from breeding and selection. Theor. Appl. Genet. 124, 1229-1240.

Sargent, D. J., Yang, Y., Šurbanovski, N., Bianco, L., Buti, M., Velasco, R., et al. (2016). HaploSNP affinities and linkage map positions illuminate subgenome composition in the octoploid, cultivated strawberry (Fragariax ananassa). Plant Sci. 242, 140150.

Schatz, M. C., Witkowski, J., and McCombie, W. R. (2012). Current challenges in de 
Strawberry Chromosome Evolution

novo plant genome sequencing and assembly. Genome Biol. 13, 243. doi:10.1186/gb-2012-13-4-243.

Schiffthaler, B., Bernhardsson, C., Ingvarsson, P. K., and Street, N. R. (2017). BatchMap: A parallel implementation of the OneMap R package for fast computation of F1 linkage maps in outcrossing species. PLoS One 12, e0189256.

Senanayake, Y. D. A., and Bringhurst, R. S. (1967). Origin of Fragaria polyploids. I. Cytological analysis. Am. J. Bot. 54, 221-228.

Shaw, D. V (1995). Comparison of ancestral and current-generation inbreeding in an experimental strawberry breeding population. Theor. Appl. Genet. 90, 237-241.

Shulaev, V., Sargent, D. J., Crowhurst, R. N., Mockler, T. C., Folkerts, O., Delcher, A. L., et al. (2011). The genome of woodland strawberry (Fragaria vesca). Nat. Genet. $43,109$.

Sjulin, T. M., and Dale, A. (1987). Genetic diversity of North American strawberry cultivars. J. Hortic. Sci. Biotechnol.

Soltis, D. E., Segovia-Salcedo, M. C., Jordon-Thaden, I., Majure, L., Miles, N. M., Mavrodiev, E. V, et al. (2014a). Are polyploids really evolutionary dead-ends (again)? A critical reappraisal of Mayrose et al.(2011). New Phytol. 202, 11051117.

Soltis, D. E., Visger, C. J., Marchant, D. B., and Soltis, P. S. (2016). Polyploidy: pitfalls and paths to a paradigm. Am. J. Bot. 103, 1146-1166.

Soltis, P. S., Liu, X., Marchant, D. B., Visger, C. J., and Soltis, D. E. (2014b). Polyploidy and novelty: Gottlieb's legacy. Philos. Trans. R. Soc. B Biol. Sci. 369, 20130351.

Spangelo, L. P. S., Hsu, C. S., Fejer, S. O., and Watkins, R. (1971). Inbred linex tester analysis and the potential of inbreeding in strawberry breeding. Can. J. Genet. Cytol. 13, 460-469.

Spigler, R. B., Lewers, K. S., Johnson, A. L., and Ashman, T.-L. (2010). Comparative mapping reveals autosomal origin of sex chromosome in octoploid Fragaria virginiana. J. Hered. 101, S107-S117.

Spigler, R. B., Lewers, K. S., Main, D. S., and Ashman, T. L. (2008). Genetic mapping of sex determination in a wild strawberry, Fragaria virginiana, reveals earliest form of sex chromosome. Heredity (Edinb). 101, 507.

Staudt, G. (1999). Systematics and geographic distribution of the American strawberry species: Taxonomic studies in the genus Fragaria (Rosaceae: Potentilleae). Univ of California Press.

Stegmeir, T. L., Finn, C. E., Warner, R. M., and Hancock, J. F. (2010). Performance of an elite strawberry population derived from wild germplasm of Fragaria chiloensis and $F$. virginiana. HortScience 45, 1140-1145.

Sun, H., Wu, S., Zhang, G., Jiao, C., Guo, S., Ren, Y., et al. (2017). Karyotype stability and unbiased fractionation in the paleo-allotetraploid Cucurbita genomes. Mol. Plant 10, 1293-1306.

Tennessen, J. A., Govindarajulu, R., Ashman, T.-L., and Liston, A. (2014). Evolutionary origins and dynamics of octoploid strawberry subgenomes revealed by dense targeted capture linkage maps. Genome Biol. Evol. 6, 3295-3313.

Tennessen, J. A., Wei, N., Straub, S. C. K., Govindarajulu, R., Liston, A., and Ashman, T.-L. (2018). Repeated translocation of a gene cassette drives sex-chromosome turnover in strawberries. PLoS Biol. 16, e2006062. 
921

922

923

924

925

926

927

928

929

930

931

932

933

934

935

936

937

938

939

940

941

942

943

944

945

946

947

948

949

950

951

952

953

954

955

956

957

958

959

960

961

962

963

964

965

966
Treangen, T. J., and Salzberg, S. L. (2012). Repetitive DNA and next-generation sequencing: computational challenges and solutions. Nat. Rev. Genet. 13, 36.

Vallejo-Marín, M., Buggs, R. J. A., Cooley, A. M., and Puzey, J. R. (2015). Speciation by genome duplication: Repeated origins and genomic composition of the recently formed allopolyploid species Mimulus peregrinus. Evolution (N. Y). 69, 1487-1500.

van Dijk, T., Pagliarani, G., Pikunova, A., Noordijk, Y., Yilmaz-Temel, H., Meulenbroek, B., et al. (2014). Genomic rearrangements and signatures of breeding in the allooctoploid strawberry as revealed through an allele dose based SSR linkage map. BMC Plant Biol. 14, 55.

VanBuren, R., Wai, C. M., Pardo, J., Yocca, A. E., Wang, X., Wang, H., et al. (2019). Exceptional subgenome stability and functional divergence in allotetraploid teff, the primary cereal crop in Ethiopia. bioRxiv, 580720.

Verma, S., Bassil, N. V, van de Weg, E., Harrison, R. J., Monfort, A., Hidalgo, J. M., et al. (2016). Development and evaluation of the Axiom ${ }^{\circledR}$ IStraw35 384HT array for the allo-octoploid cultivated strawberry Fragariax ananassa. in VIII International Strawberry Symposium 1156, 75-82.

Vining, K. J., Salinas, N., Tennessen, J. A., Zurn, J. D., Sargent, D. J., Hancock, J., et al. (2017). Genotyping-by-sequencing enables linkage mapping in three octoploid cultivated strawberry families. PeerJ 5, e3731.

Wendel, J. F., Jackson, S. A., Meyers, B. C., and Wing, R. A. (2016). Evolution of plant genome architecture. Genome Biol. 17, 37.

Yakimowski, S. B., and Rieseberg, L. H. (2014). The role of homoploid hybridization in evolution: a century of studies synthesizing genetics and ecology. Am. J. Bot. 101, 1247-1258. 


\section{Strawberry Chromosome Evolution}

\section{7}

968

969

970

971

972

973

974

975

976

977

978

979

980

981

982

983

984

985

986

987

\section{Figure Legends}

Figure 1. High-density haplotype map of a California beach strawberry ( $F$. chiloensis subsp. lucida) genome. (A) Del Norte genetic map distances plotted against the Camarosa v1.0 physical genome. Box outlines indicate groups of ancestral chromosome homoeologs. (B) Del Norte linkage groups plotted with corresponding chromosome heatmap of pairwise recombination fractions (upper diagonal) and pairwise linkage (lower diagonal).

Figure 2. Comparison of genomic heterozygosity and diploid segregation distortion in $F$. $x$ ananassa (Camarosa) and $F$. chiloensis (Del Norte) on three chromosomes (6-2, 6-4, 7-3). (A) Frequency of AA (green), AB (red), and off-target (BB; blue) genotypes for polymorphic markers in the mapping population. (B) Chi-square statistic estimating segregation distortion of polymorphic markers in the mapping population. (C) Heterozygous nucleotide frequency of parent genotypes in $20 \mathrm{~kb}$ physical windows.

Figure 3. Flowchart of bioinformatic protocols used to select genome-wide sequence variants for design of the $850 \mathrm{~K}$ SNP screening array.

Figure 4. Genetic maps of three wild octoploid strawberry genotypes (PI552277-red; PI612493-blue; Del Norte-green) based on 50K SNP array genotypes plotted against the Camarosa v1.0 physical genome. Grey highlighted chromosome segments indicate contiguous (up to $500 \mathrm{~kb}$ ) regions of the physical genome represented by the Camarosa $50 \mathrm{~K}$ SNP array map.

Figure 5. Comparative mapping of four wild octoploid Fragaria subspecies $(F$. chiloensis subsp. lucida, F. chiloensis subsp. pacifica, F. virginiana subsp. platypetala, F. virginiana subsp. virginiana) on chromosome 6-2. 


\section{Strawberry Chromosome Evolution}

1013

1014

1015

1016

\begin{tabular}{|c|c|c|c|c|c|c|}
\hline $\begin{array}{c}\text { Octoploid } \\
\text { Chromosome }\end{array}$ & $\begin{array}{c}\text { Proposed } \\
\text { Origin (Edger } \\
\text { et al., 2019) }\end{array}$ & $\begin{array}{l}\text { Spigler et } \\
\text { al. (2010) }\end{array}$ & $\begin{array}{l}\text { Tennessen } \\
\text { et al. (2014) }\end{array}$ & $\begin{array}{c}\text { van Dijk et } \\
\text { al. (2014) }\end{array}$ & $\begin{array}{l}\text { Davik et } \\
\text { al. (2015) }\end{array}$ & $\begin{array}{l}\text { Sargent et } \\
\text { al. (2016) }\end{array}$ \\
\hline $1-2$ & F. iinumae & I.D & $\mathrm{I}-\mathrm{Bi}$ & $1 \mathrm{C}$ & $1-B$ & $1 b$ \\
\hline $2-4$ & F. iinumae & II.B & II-Bi & $2 \mathrm{C}$ & 2-D & $2 b$ \\
\hline $3-2$ & F. iinumae & III.A & III-Bi & $3 D$ & $3-B$ & $3 b$ \\
\hline $4-4$ & F. iinumae & IV.C & IV-Bi & $4 \mathrm{~A}$ & $4-D$ & $4 b$ \\
\hline $5-3$ & F. iinumae & V.D & V-Bi & $5 B$ & $5-C$ & $5 b$ \\
\hline $6-3$ & F. iinumae & VI.D & $\mathrm{VI}-\mathrm{Bi}$ & $6 B$ & $6-C$ & $6 \times 2$ \\
\hline $7-3$ & F. iinumae & VII.A & VII-Bi & $7 D$ & $7-C$ & $7 b$ \\
\hline $1-3$ & F. nipponica & I.C & I-B2 & $1 \mathrm{~B}$ & $1-C$ & $1 \times 2$ \\
\hline $2-1$ & F. nipponica & II.D & II-B1 & $2 \mathrm{D}$ & $2-A$ & $2 \times 2$ \\
\hline $3-3$ & F. nipponica & III.C & III-B1 & $3 B$ & $3-C$ & $3 \times 2$ \\
\hline $4-2$ & F. nipponica & IV.D & IV-B2 & $4 \mathrm{D}$ & 4-B & $4 \times 2$ \\
\hline $5-4$ & F. nipponica & V.B & V-B2 & $5 D$ & 5-D & $5 \times 1$ \\
\hline $6-2$ & F. nipponica & VI.B & VI-B1 & $6 \mathrm{D}$ & $6-B$ & $6 b$ \\
\hline $7-1$ & F. nipponica & VII.D & VII-B2 & $7 C$ & $7-A$ & $7 X 2$ \\
\hline $1-4$ & F. vesca & I.A & $\mathrm{I}-\mathrm{Av}$ & $1 \mathrm{~A}$ & 1-D & $1 \mathrm{~A}$ \\
\hline $2-2$ & F. vesca & II.A & II-Av & $2 \mathrm{~A}$ & $2-B$ & $2 \mathrm{~A}$ \\
\hline $3-4$ & F. vesca & III.D & III-Av & $3 A$ & $3-D$ & $3 A$ \\
\hline $4-3$ & F. vesca & IV.A & IV-Av & $4 B$ & $4-C$ & $4 \mathrm{~A}$ \\
\hline $5-1$ & F. vesca & V.C & V-Av & $5 A$ & $5-A$ & $5 A$ \\
\hline $6-1$ & F. vesca & VI.A & VI-Av & $6 \mathrm{~A}$ & $6-A$ & $6 \mathrm{~A}$ \\
\hline $7-2$ & F. vesca & VII.B & VII-Av & $7 A$ & 7-B & $7 \mathrm{~A}$ \\
\hline $1-1$ & F. viridis & I.B & I-B1 & $1 \mathrm{D}$ & $1-A$ & $1 \mathrm{X} 1$ \\
\hline $2-3$ & F. viridis & II.C & II-B2 & $2 B$ & $2-C$ & $2 \times 1$ \\
\hline $3-1$ & F. viridis & III.B & III-B2 & $3 C$ & $3-A$ & $3 \times 1$ \\
\hline $4-1$ & F. viridis & IV.B & IV-B1 & $4 C$ & $4-A$ & $4 \times 1$ \\
\hline $5-2$ & F. viridis & V.A & V-B1 & $5 C$ & $5-B$ & $5 \times 2$ \\
\hline $6-4$ & F. viridis & VI.C & VI-B2 & $6 C$ & 6-D & $6 \times 1$ \\
\hline $7-4$ & F. viridis & VII.C & VII-B1 & $7 B$ & 7-D & $7 X 1$ \\
\hline
\end{tabular}

Table 1. Published octoploid strawberry linkage group nomenclatures anchored to corresponding physical chromosomes in the Camarosa v1.0 reference genome. 

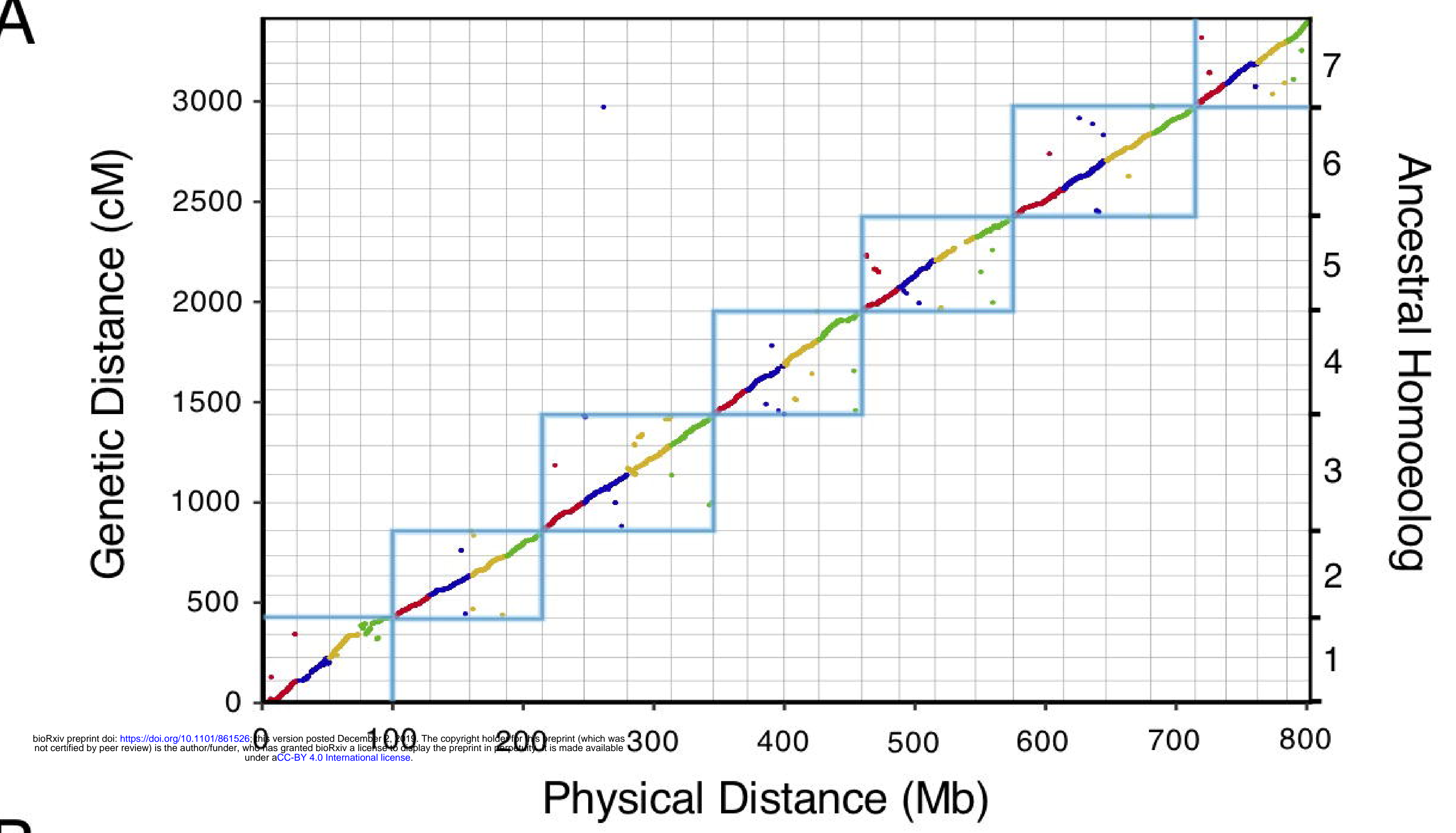

- 1-1

- 4-3

- 1-2

- 4-4

- 1-3

- 5-1

- 1-4

- 5-2

- 2-1

- 5-3

- 2-2

- 5-4

- 2-3

- 6-1

- 2-4

- 6-2

- 3-1

- 6-3

- 3-2

- 6-4

- 3-3

- 7-1

- 3-4

- 7-2

- 4-1

- 7-3

- 4-2

- 7-4

B

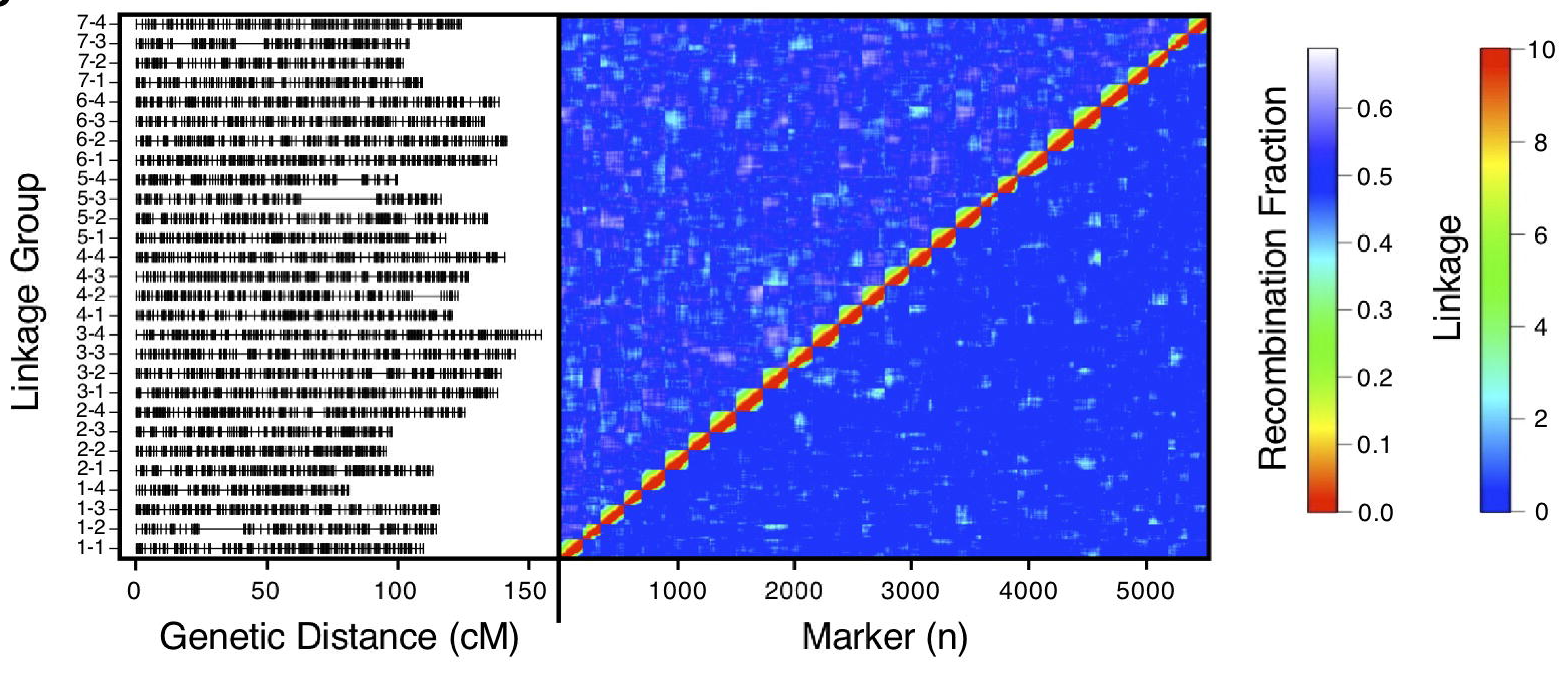




\section{6-2}

A

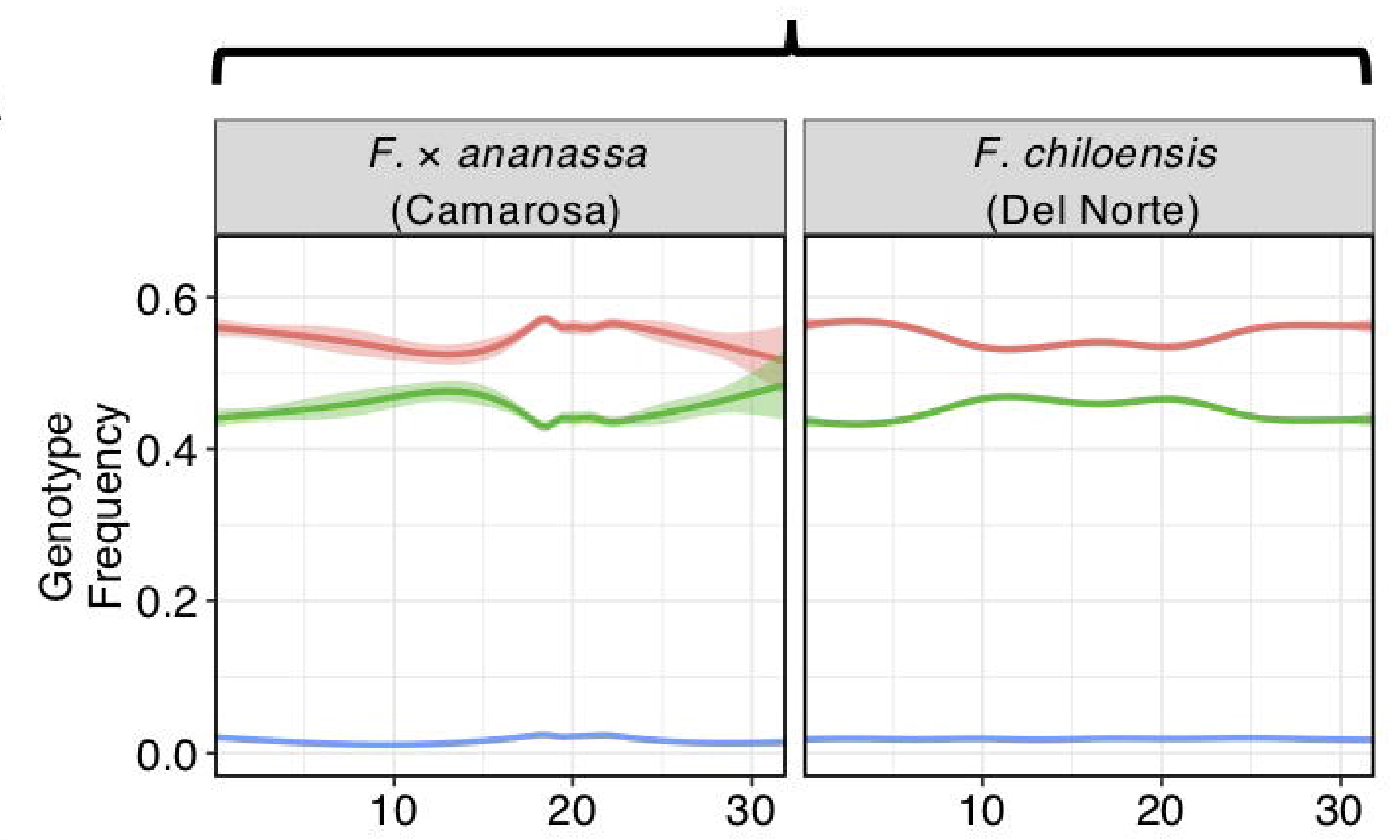

B

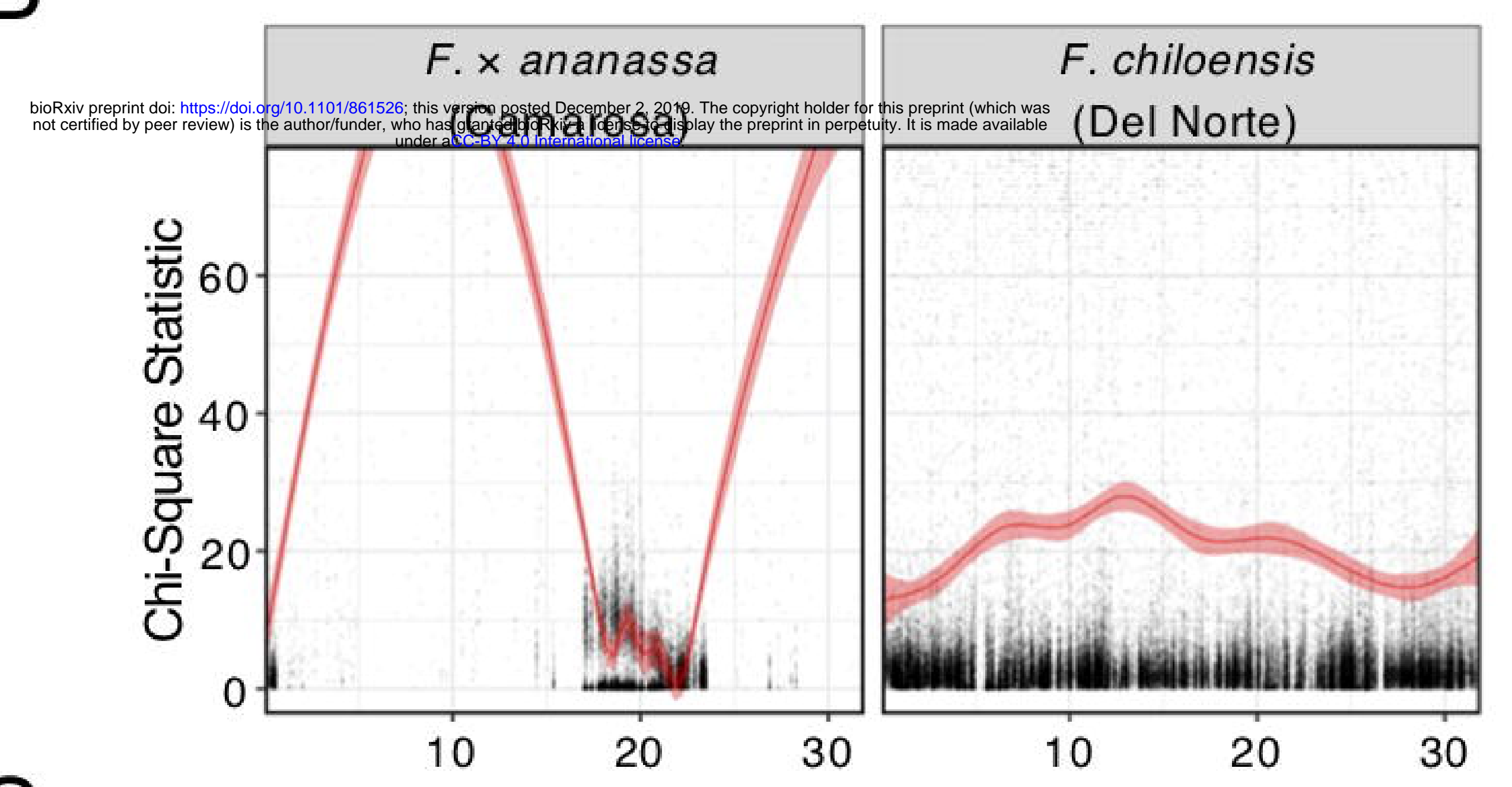

C

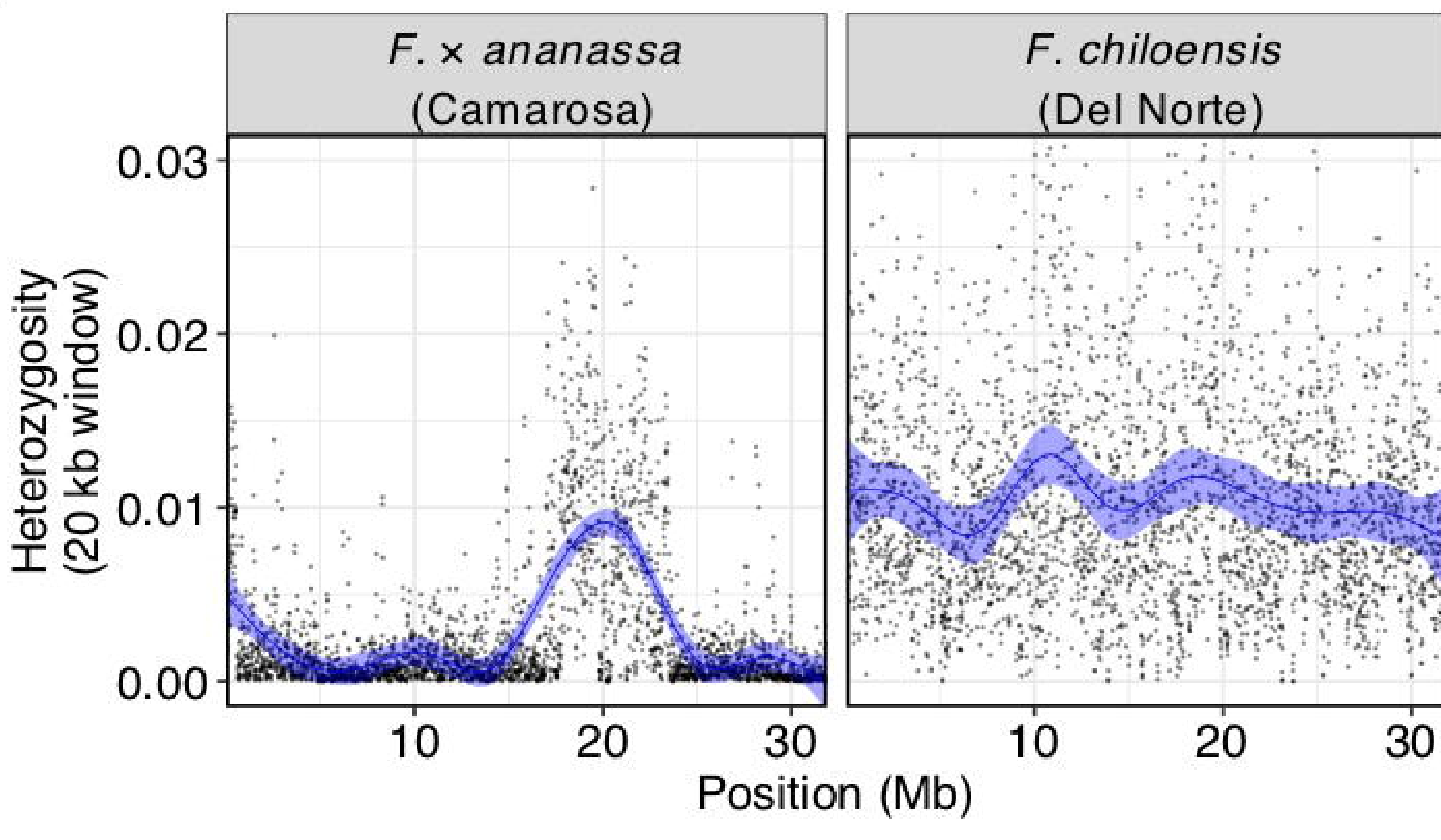

6-4
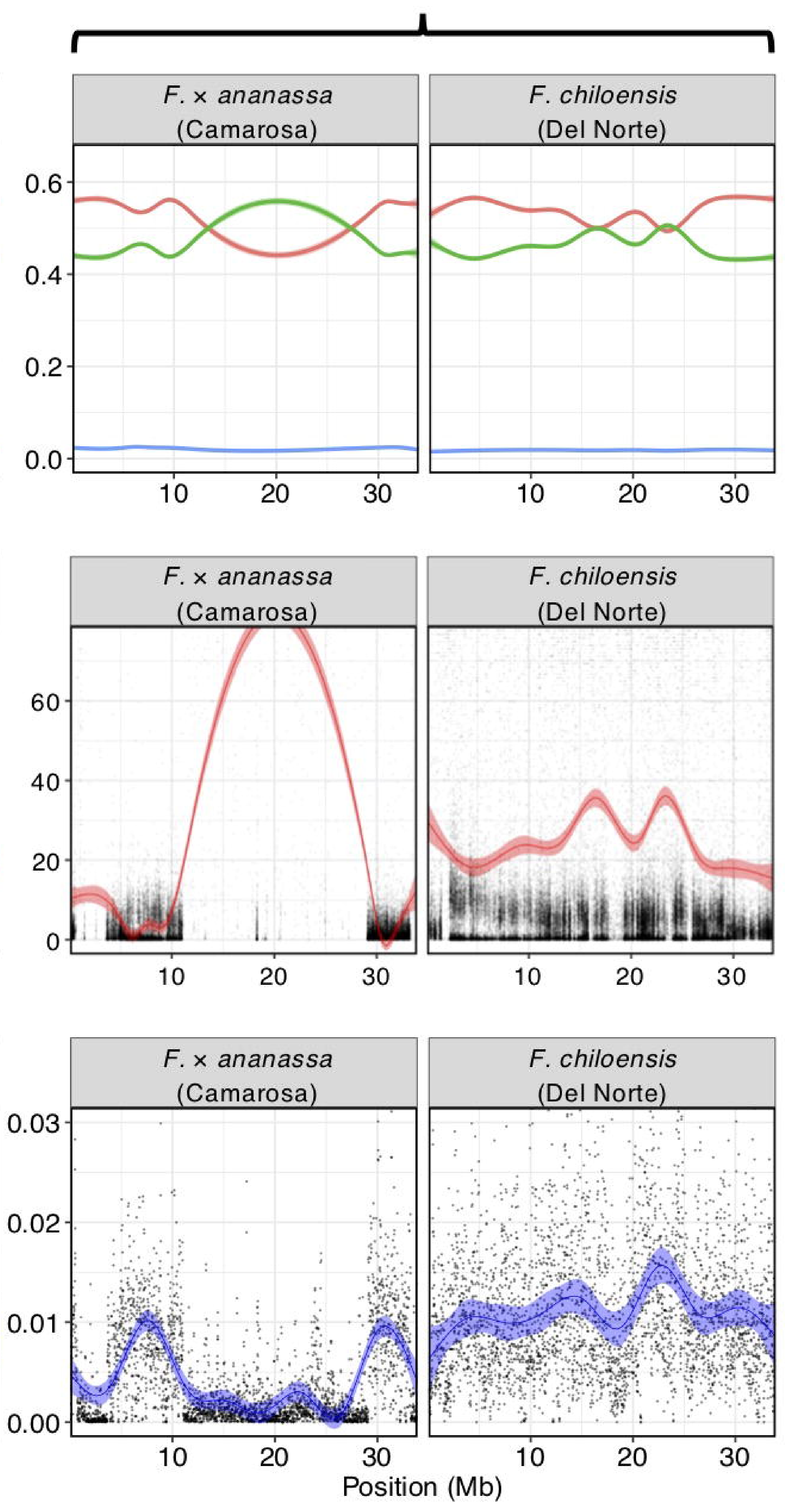

7-3
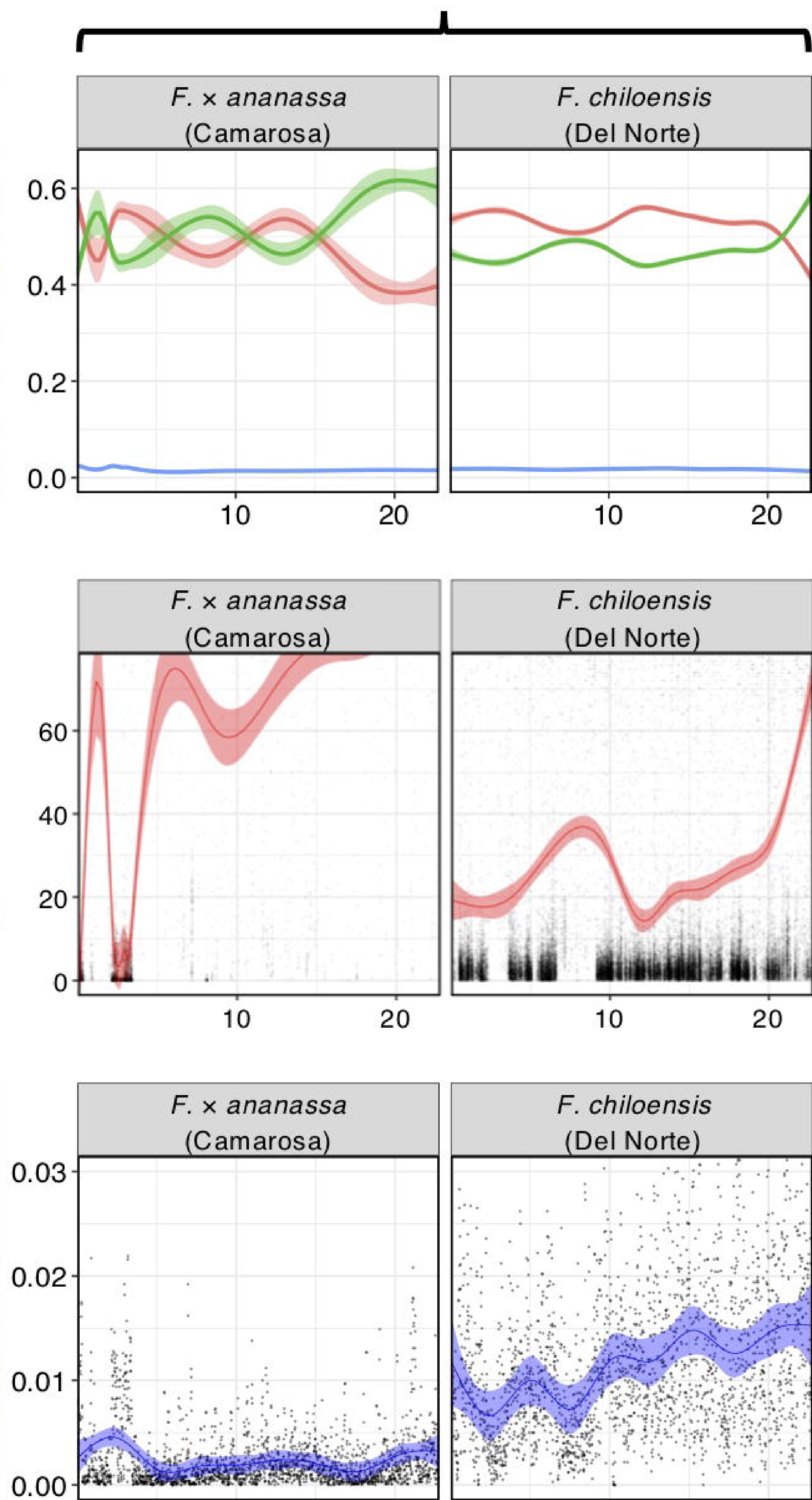

10

F. chiloensis (Del Norte)

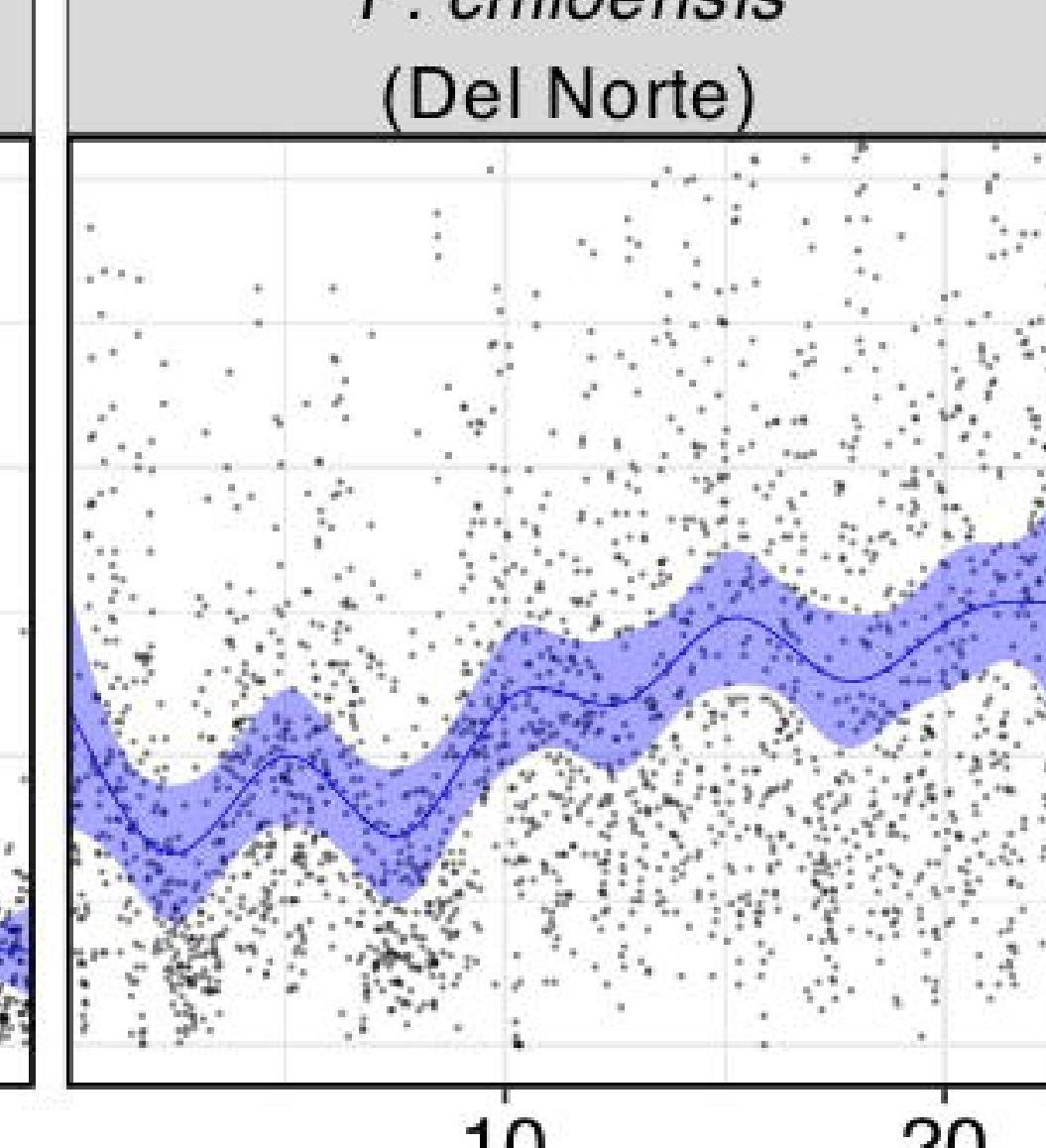




\section{Octoploid Genome Assembly}

F. $\times$ ananassa cv. 'Camarosa' V1

Assembly Size $=810 \mathrm{Mb}$

Number of Annotated Genes $=108,000$

Gene Space $=42 \%$

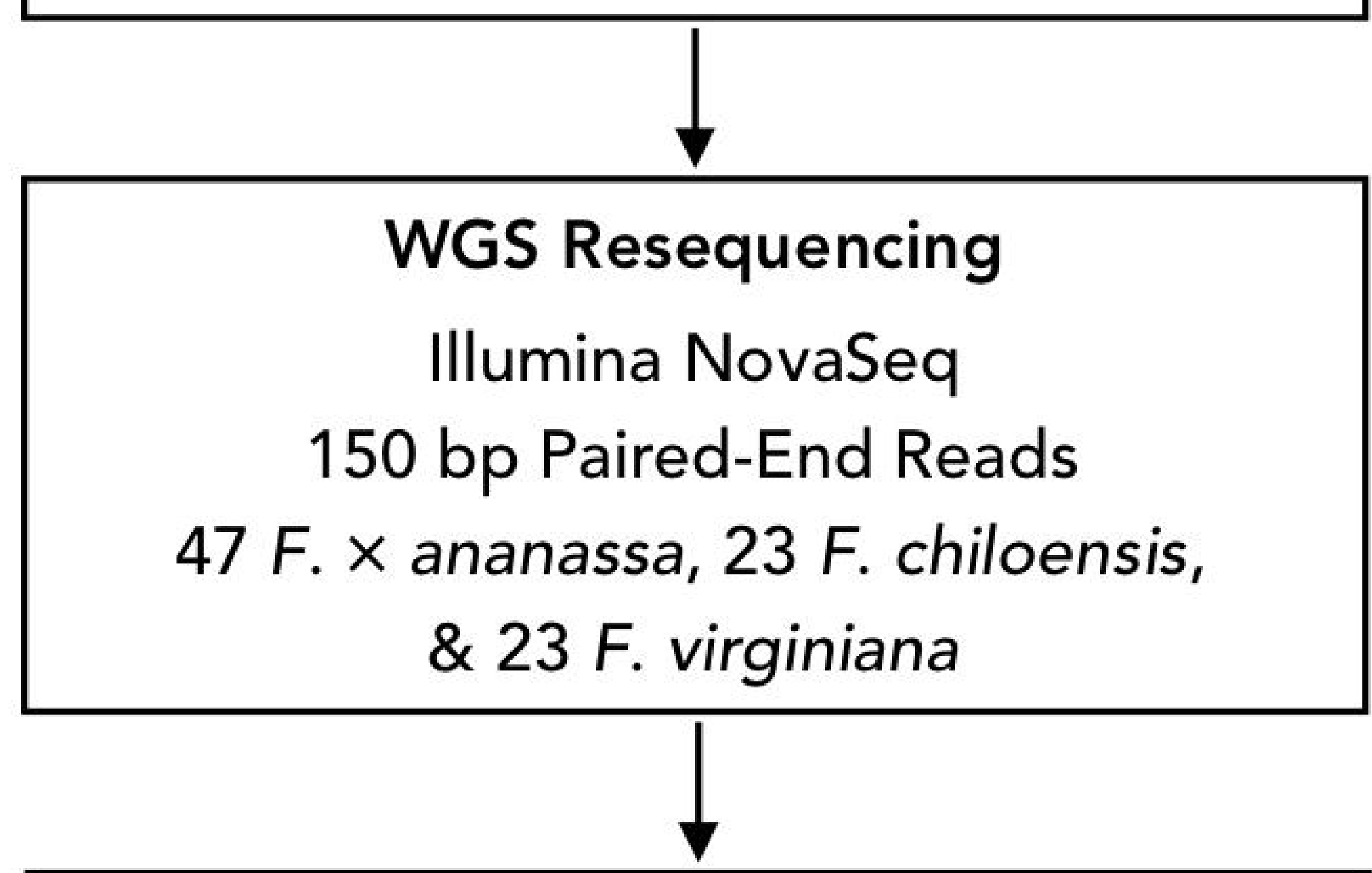

Physical Mapping \& DNA Variant Calling

Short-Read Mapping: BWA-MEM

DNA Variant Calling: FreeBayes

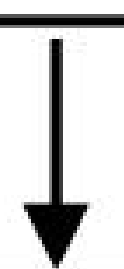

90M Unfiltered DNA Variants

SNPs, INDELs, \& Complex bioRxiv preprint doi: htpps:/doi. org/10.1101/861526. this version posted December 2, 2019. The copyright holder for this prepprint (which was $\downarrow$

45M Filtered F. $x$ ananassa DNA Variants

F. $x$ ananassa SNPs

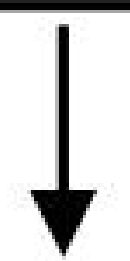

DNA Variant Filtering Round $1 \rightarrow 8.0 \mathrm{M}$ SNPs

Bi-Allelic SNPs Only MAF $>0.05$

Nucleotide Diversity $>0.05$

DNA Variant Quality Score $>20$ $<15 \%$ Missing Data
DNA Variant Filtering Round $2 \rightarrow 6.6 \mathrm{M}$ SNPs

No $\mathrm{A} / \mathrm{T}$ or $\mathrm{C} / \mathrm{G}$ (Requires 2 Probes)

No Ambiguous Sequence (Ns) in the

Probe Region

No Identical Probe Sequences

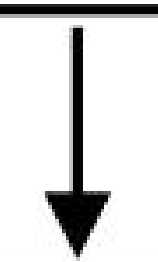

Affymetrix Probe Selection

Probe Uniqueness (kmer)

Buildability Statistics

Technical Recommendation: Yes/Neutral/

No \& Plus/Minus Strand

\section{Probe Interference Analysis}

Number of DNA Variants in 35-nt

Window

Quantification of Local Hybridization Interference: Sum of MAFs for Local Interfering DNA Variants

\section{Predicted Octoploid Sub-Genome} Specificity

Alignment of 71-nt SNP Probes to the Octoploid Reference Genome Quantified the Number of Off-Target Alignments: Perfect Matches, 95\% Identity Over $95 \%$ of Probe Sequence, \& $90 \%$ Probe Identity Over $90 \%$ of Probe Sequence

Selection of Probes for the 850K SNP Genotyping Array

Heterozygosity, Genome Distribution, and Quality Metrics

Included 16K iStraw35 Probes for Cross-Referencing 


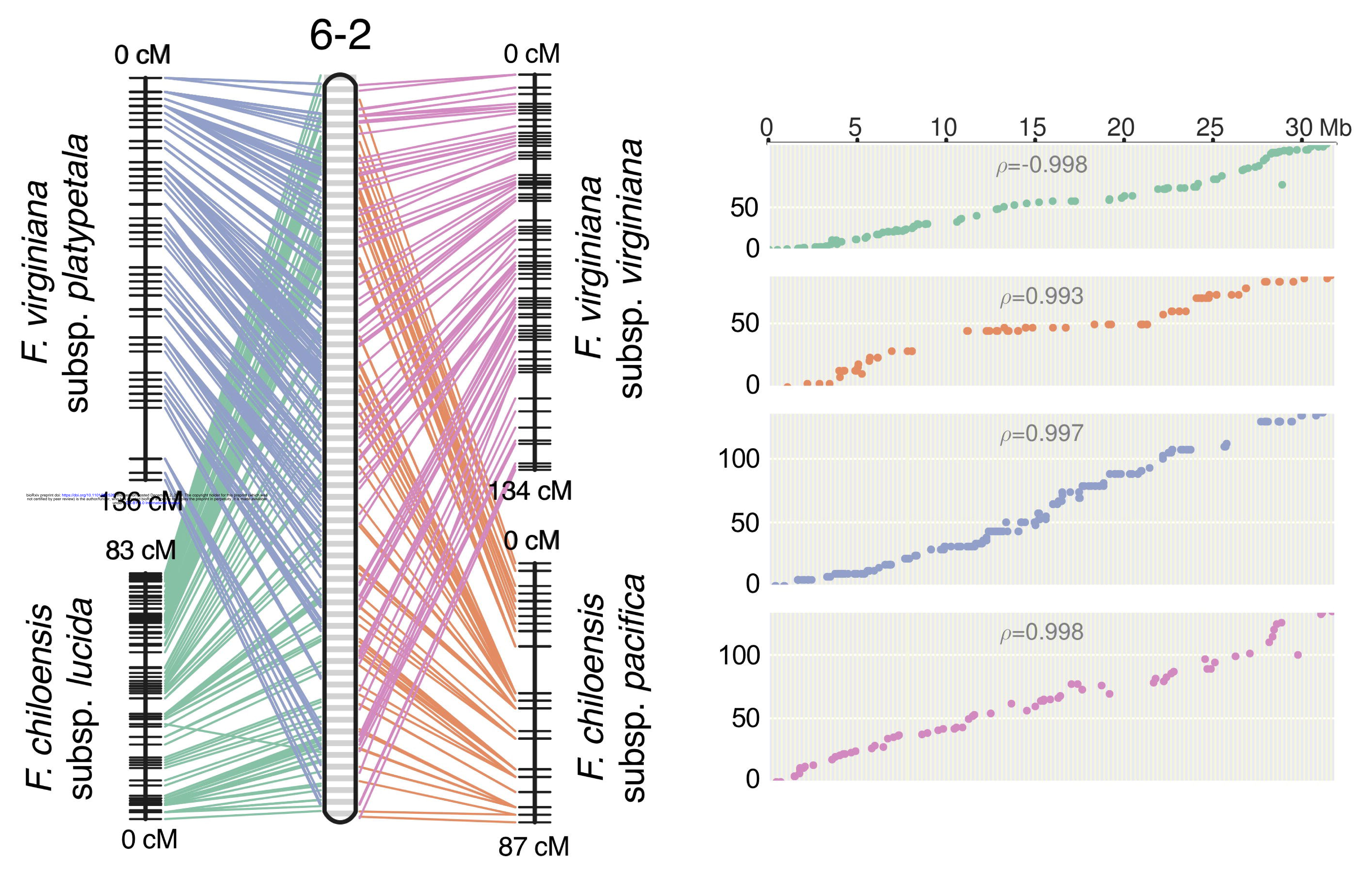

